\title{
Early post-zygotic mutations contribute to congenital heart disease
}

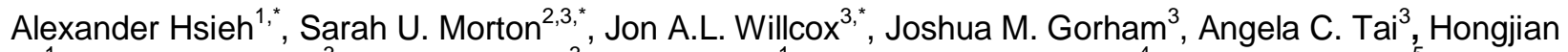
Qi ${ }^{1}$, Steven DePalma ${ }^{3}$, David McKean ${ }^{3}$, Emily Griffin ${ }^{1}$, Kathryn B. Manheimer ${ }^{4}$, Daniel Bernstein ${ }^{5}$, Richard W. Kim $^{6}$, Jane W. Newburger ${ }^{2}$, George A. Porter Jr. ${ }^{7}$, Deepak Srivastava ${ }^{8}$, Martin Tristani-Firouzi ${ }^{9}$, Martina Brueckner ${ }^{10}$, Richard P. Lifton ${ }^{14}$, Elizabeth Goldmuntz ${ }^{13}$, Bruce D. Gelb ${ }^{4}$, Wendy K. Chung ${ }^{1, \#,}$ Christine E. Seidman ${ }^{3,11,12 \#}$, J. G. Seidman ${ }^{3, \#}$, Yufeng Shen ${ }^{1, \#^{n}}$

${ }^{1}$ Columbia University Medical Center, New York, NY, USA

${ }^{2}$ Boston Children's Hospital, Boston, Massachusetts, USA.

${ }^{3}$ Harvard Medical School, Boston, Massachusetts, USA.

${ }^{4}$ Icahn School of Medicine at Mount Sinai, New York, New York, USA

${ }^{5}$ Stanford University, Palo Alto, California, USA.

${ }^{6}$ Children's Hospital Los Angeles, Los Angeles, California, USA.

${ }^{7}$ University of Rochester Medical Center, Rochester, New York, USA.

${ }^{8}$ Gladstone Institutes and University of California San Francisco, San Francisco, California, USA.

${ }^{9}$ University of Utah School of Medicine, Salt Lake City, Utah, USA.

${ }^{10}$ Yale University School of Medicine, New Haven, Connecticut, USA.

${ }^{11}$ Brigham and Women's Hospital, Boston, Massachusetts, USA

12 Howard Hughes Medical Institute, Harvard University, Boston, Massachusetts, USA

${ }_{13}^{13}$ Children's Hospital of Philadelphia, Philadelphia, Pennsylvania, USA

${ }^{14}$ Rockefeller University, New York, NY, USA

${ }^{*}$, \# Equal contribution.

'Corresponding author. Email: ys2411@cumc.columbia.edu (Y.S.).

alh2194@cumc.columbia.edu (A.H.)

Sarah.Morton@childrens.harvard.edu (S.U.M.)

Jon Willcox@hms.harvard.edu (J.A.L.W.)

igorham@genetics.med.harvard.edu (J.M.G.)

angela tai@hms.harvard.edu (A.C.T.)

hq2130@columbia.edu (H.Q.)

depalma@genetics.med.harvard.edu (S.D.)

dmckean@genetics.med.harvard.edu (D.M.)

eg2871@cumc.columbia.edu (E.G.)

kathryn.manheimer@icahn.mssm.edu (K.B.M.)

danb@stanford.edu (D.B.)

rikim@chla.usc.edu (R.W.K.)

jane.newburger@cardio.chboston.org (J.W.N.)

george porter@urmc.rochester.edu (G.A.P.)

deepak.srivastava@gladstone.ucsf.edu (D.S.)

martin.tristani@utah.edu (M.T.F.)

martina.brueckner@yale.edu (M.B.)

rickl@mail.rockefeller.edu (R.P.L.)

Goldmuntz@email.chop.edu (E.G.)

bruce.gelb@mssm.edu (B.D.G.)

wkc15@cumc.columbia.edu (W.K.C.)

cseidman@genetics.med.harvard.edu (C.E.S.)

seidman@genetics.med.harvard.edu (J.G.S.)

ys2411@cumc.columbia.edu (Y.S.) 


\section{Abstract}

\section{Background}

55 The contribution of somatic mosaicism, or genetic mutations arising after oocyte fertilization, to congenital

56 heart disease (CHD) is not well understood. Further, the relationship between mosaicism in blood and

57 cardiovascular tissue has not been determined.

Results

60 We developed a computational method, Expectation-Maximization-based detection of Mosaicism (EM-

61 mosaic), to analyze mosaicism in exome sequences of $2530 \mathrm{CHD}$ proband-parent trios. EM-mosaic

62 detected 326 mosaic mutations in blood and/or cardiac tissue DNA. Of the 309 detected in blood DNA,

$6385 / 97$ (88\%) tested were independently confirmed, while $7 / 17$ (41\%) candidates of 17 detected in cardiac

64 tissue were confirmed. MosaicHunter detected an additional 64 mosaics, of which 23/46 (50\%) among 58

65 candidates from blood and 4/6 (67\%) of 6 candidates from cardiac tissue confirmed. Twenty-five mosaic

66 variants altered CHD-risk genes, affecting $1 \%$ of our cohort. Of these $25,22 / 22$ candidates tested were

67 confirmed. Variants predicted as damaging had higher variant allele fraction than benign variants,

68 suggesting a role in CHD. The frequency of mosaic variants above $10 \%$ mosaicism was $0.13 /$ person in

69 blood and 0.14/person in cardiac tissue. Analysis of 66 individuals with matched cardiac tissue available

70 revealed both tissue-specific and shared mosaicism, with shared mosaics generally having higher allele

71 fraction.

\section{Conclusions}

74 We estimate that $\sim 1 \%$ of $\mathrm{CHD}$ probands have a mosaic variant detectable in blood that could contribute

75 to cardiac malformations, particularly those damaging variants expressed at higher allele fraction

76 compared to benign variants. Although blood is a readily-available DNA source, cardiac tissues analyzed

77 contributed $\sim 5 \%$ of somatic mosaic variants identified, indicating the value of tissue mosaicism analyses.

79 Keywords

80 Mosaic, Somatic, Congenital Heart Disease, Exome Sequencing 


\section{Background}

Mosaicism results from somatic mutations that arise post-zygotically in an early embryonic cell,

83 resulting in two or more cell populations with distinct genotypes in the developing embryo \{Biesecker

$842013\}$. The developmental status of the early embryonic cell at the time of mutagenesis determines the proportion of variant-carrying cells and the tissue distribution of these cells in the post-natal child \{AcunaHidalgo 2015\}. While germline variants have a variant allele frequency (VAF) of 0.5 , somatic mosaic variants have a significantly lower VAF.

Post-zygotic mosaic mutations have been implicated in several diseases including non-malignant

89 developmental disorders such as overgrowth syndromes \{Poduri 2013; Lindhurst 2012; Kurek 2016\},

90 structural brain malformations \{Poduri 2012; Jamuar 2014; Riviere 2012; Lee 2012\}, epilepsy \{Stosser

$912018\}$, and autism spectrum disorder \{Lim 2017; Krupp 2017; Freed 2016; Dou 2017\}. Recent analyses

92 also identified mosaic variants in a cohort of patients with congenital heart disease (CHD) \{Manheimer

$932018\}$, but the prevalence of these was far less than germline variants (CHD) \{Zaidi 2013; Homsy 2015;

94 Jin 2017; Zaidi 2017\}.

95 Assessment of the frequency of mosaicism in human disease is confounded by technical issues,

96 including differences in sequencing depth, DNA sources, and variant assessment pipelines. Low levels of

97 mosaicism can escape the detection threshold of traditional sequencing methods with standard read

98 depths, while post-zygotic mutations with a higher percentage of affected cells are difficult to discriminate

99 from germline de novo mutations \{Acuna-Hidalgo 2015\}. All of these issues can lead to substantially

100 different conclusions. For example, analyses of mosaicism in autism spectrum disorder was recently

101 assessed from whole exome sequence (WES) data from whole blood DNA from 2506 families (proband,

102 parents and unaffected sibling; trios and quads) in the Simons Simplex Collection (SSC) \{Fischbach

$1032010\}$. The primary sequence data were analyzed by three groups; one that identified a protein-coding

104 somatic mosaic variant rate of 0.074 per individual \{Freed 2016\}, another that found a mosaic rate of

1050.059 per individual $\{$ Lim 2017\}, and a third group that reported a mosaic rate of 0.125 per individual

$106\{$ Krupp 2017\}. This disparity suggests the need for more systematic mosaic mutation detection methods

107 that account for dataset-specific confounding factors. 
By contrast, analyses of affected tissues can improve the sensitivity and specificity of detection of

109 somatic mosaicism. In cancer, methods to detect these events, such as MuTect \{Cibulskis 2013\},

110 compare tumor and benign tissues from the same patient. Mosaicism has also been demonstrated from

111 the analyses of unpaired samples with cancer and other pathologies \{Sun 2018; Huang 2017; Smith

$1122015\}$ by the demonstration of variants in affected tissues that are absent from blood-derived DNA

113 \{Symoens 2017; McDonald 2018\}. With access to cardiac tissues from patients with CHD obtained during

114 surgical repair, we hypothesized that analyses of mosaicism in cardiac tissue might improve insights into

115 the causes of this common congenital anomaly. As many cardiomyocyte lineages share a mesodermic

116 origin with blood cells but exit the cell cycle during embryogenesis, we also sought to determine if

117 mosaicism in the heart exhibited distinct patterns of mosaicism with regard to variant frequency and allele 118 fractions.

119 In this study, we developed a computational method, EM-mosaic (Expectation-Maximization-

120 based detection of Mosaicism), to detect mosaic single nucleotide variants (SNVs) using WES of proband

121 and parent DNA. To optimize this method, we measured mosaic detection power as a function of

122 sequencing depth. We compared EM-mosaic and MosaicHunter \{Huang 2014\} to investigate mosaicism

123 in 2530 CHD proband-parent trios from the Pediatric Cardiac Genomics Consortium (PCGC) \{Jin et al

1242017 , using exome sequences derived from blood-derived DNA. We detected predicted deleterious

125 mosaic mutations in genes involved in known biological processes relevant to CHD or developmental

126 disorders in $1 \%$ of probands. The accuracy of these mosaic variant detection algorithms was assessed

127 using an independent re-sequencing method. We found that among high-confidence mosaic mutations in

128 CHD-relevant genes, likely-damaging variants tended to have higher VAF than likely-benign variants.

129 In parallel we assessed mosaicism by EM-mosaic and MosaicHunter in 70 discarded tissues from

130 several heart regions obtained from 66 probands who underwent cardiac surgical repairs. While VAF

131 varied significantly ( $>3$ fold) between blood and cardiovascular tissue at about $60 \%$ of sites, in general

132 mosaic variants with high (>15\%) VAF were more likely shared between blood and cardiac tissue than

133 variants with lower VAF.

\section{Results}


High-accuracy detection of mosaic mutations in WES data using EM-mosaic

We analyzed whole exome sequence (WES) data from $2530 \mathrm{CHD}$ proband-parent trios \{Homsy 2015; Jin 2017\} (Table S1). Among this cohort, 1205 probands had CHD with neurodevelopmental disorders (NDD) and/or extracardiac manifestations (EM), 788 had isolated CHD at the time of enrollment, 539 had undetermined NDD status due to young neonatal age at the time of enrollment, and 9 subjects had incomplete data (Table S2).

Previous WES analyses \{Jin et al 2017\} identified 1742 germline de novo SNVs among 838 cases with NDD and/or EM, 516 isolated cases, 644 cases of unknown NDD status, and 7 with

144 incomplete data. These de novo variants were identified using the Genome Analysis Toolkit (GATK) 145 pipeline \{McKenna 2010; DePristo 2011\} assuming a germline diploid model in which the expected VAF 146 is 0.5 . This model has limited sensitivity to detect mosaic mutations for which the fraction of alternative 147 allele reads is significantly below 0.5 , especially because de novo variants with $\mathrm{VAF}<0.2$ were excluded 148 to reduce false discovery.

To efficiently capture mosaic variants with $\mathrm{VAF}<0.4$, we developed a new method (EM-mosaic) to

150 detect mosaic variants in WES sequence of a proband and parents (trios). Potential mosaic variants were 151 identified in WES sequence data using SAMtools mpileup $\{\mathrm{Li} 2009\}$ with settings designed to capture 152 sites with VAF between 0.1-0.4 and merged with the variants found by the GATK pipeline \{Jin et al 2017\}

153 (Fig S1) to create a union variant set. To reduce the elevated false positive rate inherent in low-VAF calls, 154 we applied a set of empirical filters to remove likely technical artifacts due to sequencing errors associated with repetitive and/or low complexity sequences. We then manually inspected de novo SNVs

156 with $\mathrm{VAF}<0.3(\mathrm{n}=582)$ using IGV and filtered out an additional 188 likely false positives. After

157 preprocessing and outlier removal, the remaining 2971 de novo SNVs were used as input to our mosaic 158 detection model.

Among the 2971 de novo SNVs, this pipeline identified 309 sites as candidate mosaics based on

160 posterior odds score (Fig 1A-B, Table S3), including 50 sites that were previously reported as germline 161 de novo variants \{Jin et al 2017\}. An additional 86 sites were identified as having posterior odds below 162 our threshold of 10 but greater than 1 (Fig S2A, S2B), including a ZEB2 variant with posterior odds 4.7 163 that was previously confirmed via ddPCR \{Manheimer 2018\}. Among these 86 variants, 53 are likely 
164 mosaic and 33 are likely germline (Fig S2B). We chose not to include these sites since there was

165 insufficient evidence to confidently resolve them individually as mosaic or germline.

167 Mosaic mutations found in blood derived DNA with MosaicHunter

168 We also employed MosaicHunter, which uses a Bayesian genotyping algorithm with a series of

169 stringent filters (see Supplemental Methods) for discovering mosaic variants using WGS genotype

170 information from trios. \{Huang 2017\} Among the $2530 \mathrm{CHD}$ trios, MosaicHunter identified an initial set of

17158976 sites showing evidence of mosaicism, including 214 high-confidence variants located in coding

172 regions. (Fig S3). After applying a minimum likelihood ratio (LR) cutoff of 80 for distinguishing mosaic

173 from germline mutation, and additional heuristic filters (Supplemental Methods), MosaicHunter identified

174116 coding sites (Table S4) or 0.05 mosaics /individual.

175 Of the mosaic candidates detected by MosaicHunter, 58/116 (50\%) were also identified by EM-

176 mosaic while 58/116 (50\%) candidates were unique to MosaicHunter (Table 1; Fig S4). Of the 58

177 candidates unique to MosaicHunter, 35 were filtered out by EM-mosaic on the basis of insufficient

178 alternate allele read support, 16 had a non-zero allelic depth in the parents, and 7 failed quality filters.

179 The 251 candidates unique to EM-mosaic were discarded by the MosaicHunter pipeline during BAM

180 reprocessing $(n=13)$, quality filtering $(n=146)$, application of LR cutoff $(22)$, or were not called due to

181 inadequate read depth ( $\mathrm{n}=70)$ (Fig S3).

182

Table 1: Mosaic variant detection by EM-Mosaic, MosaicHunter and validated by PCR product sequencing

\begin{tabular}{|c|c|c|c|c|c|}
\hline \multirow{2}{*}{\multicolumn{2}{|c|}{ 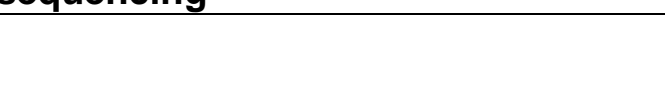 }} & \multirow[t]{2}{*}{ Union } & \multirow[t]{2}{*}{ Shared } & \multicolumn{2}{|c|}{ Unique } \\
\hline & & & & EM-Mosaic & MosaicHunter \\
\hline \multicolumn{2}{|c|}{ Mosaic Variants (total)* } & $315(332)$ & $56(57)$ & $218(240)$ & $29(35)$ \\
\hline \multicolumn{2}{|c|}{ Mosaic Candidates } & 367 & 58 & 251 & 58 \\
\hline \multicolumn{2}{|c|}{ Mosaic Candidate VAF mean (SD) } & $0.13(0.06)$ & $0.12(0.05)$ & $0.13(0.06)$ & $0.10(0.05)$ \\
\hline \multirow{4}{*}{$\begin{array}{c}\text { MiSeq } \\
\text { Confirmation }\end{array}$} & Total Tested & 143 & 22 & 75 & 46 \\
\hline & Mosaic & 108 & 21 & 64 & 23 \\
\hline & Germline & 3 & 0 & 3 & 0 \\
\hline & No Variant & 32 & 1 & 8 & 23 \\
\hline \multicolumn{2}{|c|}{ Validation Rate } & $76 \%$ & $95 \%$ & $85 \%$ & $50 \%$ \\
\hline
\end{tabular}

183 *Estimated number of mosaic variants found among $2530 \mathrm{CHD}$ probands (total number of mosaic variants detected 184 by EM-mosaic and MosaicHunter). 
Sequence confirmation of candidate mosaic variants and estimation of mosaicism in CHD

From the 367 high-confidence EM-mosaic and/or MosaicHunter SNVs, we selected 143 candidates (97 identified by EM-mosaic; 68 identified by MosaicHunter) for experimental confirmation using MiSeq amplicon resequencing (Table S5; Table S11 and S12; Methods). DNA fragments encompassing the putative mosaic variant were PCR-amplified from proband and each parent DNA, sequenced on an Illumina MiSeq next generation sequencer and VAF was calculated for each individual. These candidate mosaics included SNVs on the extremes of the VAF spectrum, as well as mosaics that were flagged by MosaicHunter quality filters. Candidates mosaic variants were considered confirmed by MiSeq analyses if they demonstrated an amplicon VAF exceeding 0.01 but less than 0.45 , so as to indicate a variant of post-zygotic origin. MiSeq VAF values closely correlated with those originally determined by exome sequencing $\left(P=2.2 \times 10^{-16}\right.$; Fig S5).

We confirmed 85/97 (88\%) EM-mosaic candidate mosaic variants. Three candidate variants were

199 likely germline de novo SNVs (VAF>0.45). Nine candidate variants were 'false positives' that were neither 200 germline de novo SNVs or mosaic SNVs since either no variant reads were detected by MiSeq

201 sequencing of the proband amplicon, or the same small fraction of variants were detected in proband 202 amplicon and one parent's amplicon.

204 were 23 sites for which no variant reads were detected by MiSeq amplicon sequencing (MiSeq

$205 \mathrm{VAF}<0.001$ ) or in which the same small fraction of variant reads was detected in the proband amplicon as 206 in one parent's amplicon.

207 We considered whether estimates of mosaic variant frequency were sensitive to whole exome 208 sequencing depth by calibrating estimates of mosaic detection power using properties of the sequence 209 data (average read depth, prior mosaic fraction, and the value of our overdispersion parameter $\theta$ ) (Fig 210 S6; Supplemental Methods). Our projected mosaic detection power curves demonstrated more than a 211 doubling of power to detect mosaic variants with VAF 0.2 as sequencing depth increases from $40 x$ to $80 x$ 
212 (Fig 1C). Projected mosaic detection power curves for less stringent mosaic cutoffs showed similar

213 increases of power with increasing sequencing depth (Fig S8).

214 To estimate the 'true' frequency of mosaicism per blood DNA exome, independent of average

215 coverage detection power constraints, we estimated the 'true' mosaic count in a VAF range by multiplying

216 the number of mosaics by the inverse of the detection power for each VAF bin. Applying this method to

217 the 184 of 309 high-confidence EM-mosaic variants with VAF>0.1, we estimated the adjusted number of

218 mosaics with VAF>0.1 to be 361 (Fig S8A). Thus, the true frequency of coding mosaics in the blood

$219(0.4>V A F>0.1)$ is 0.14 variants per individual, representing a non-negligible class of mutations with

220 potential contribution to genetic risk for congenital heart disease. The estimated true mosaic frequency

221 does not change significantly when using less stringent mosaic definitions (Figure S8). In sum, we

222 identified 315 blood mosaic variants in $2530 \mathrm{CHD}$ probands or 0.13 mosaic variants per subject with a

223 mean VAF of $0.13 \pm 0.06$. We do not anticipate that doubling the sequencing depth would change

224 significantly this estimate.

Mosaic variants occurred most frequently at CpG sequences.

Previous studies demonstrated a strong preference for de novo $\mathrm{C}>\mathrm{T}$ mutations at $\mathrm{CpG}$

dinucleotides compared to other dinucleotides due to the spontaneous deamination of 5-methylcytosine

229 \{Fryxell 2005; Francioli 2015\}. We asked whether the germline de novo variants observed in CHD

230 probands and the 332 mosaic sites demonstrated a similar sequence preference (Fig 1, Table 1, S3, S4).

231 Of the 2662 germline de novo mutations identified in $2530 \mathrm{CHD}$ probands, 979 variants ( $37 \%$ of all

232 variants) involved mutation of the cytosine of a CpG dinucleotide (Fig 2A). By contrast, 99 (29\% of all

233 mosaic SNVs) of 332 mosaic SNVs altered the cytosine of a CpG dinucleotide; significantly more than

234 expected by chance $\left(2.2 \times\right.$ above expectation; $\left.\mathrm{p}=2 \times 10^{-15}\right)$. These observations suggest that somatic de

235 novo mutations were 1.4-fold less likely to involve a $\mathrm{CpG}$ dinucleotide than germline de novo mutations in

$236 \mathrm{CHD}$ probands $(P=0.01 ;$ Fig 2B). Even ignoring the high $\mathrm{CpG}$ mutation frequency, cytosines and

237 guanines were $\sim 2$-fold more likely to be mutated than adenines or thymidines both for germline mutations

238 and for mosaic variants. Surprisingly, somatic mutations of $A>C / T>G$ transversions in $A p C$ dinucleotides

239 were $\sim 2$-fold greater than the corresponding germline mutations $\left(P=5 \times 10^{-8} ;\right.$ Fig 2B). 
Detection of mosaic mutations in CHD tissues

Using EM-mosaic and MosaicHunter we analyzed exome sequences from 70 cardiac tissues derived from 66 subjects with CHD (Table S6) and paired blood samples. Among 57 de novo variants

244 (allele depth approximately 0.5) that were previously identified in blood-derived DNA, 54 were also found 245 in CHD tissues. Of the 3 de novo variants not present in cardiac tissue, 1 was outside of the tissue WES 246 capture region and 2 occurred in a single proband (Table 2). In addition, 23 distinct candidate mosaic 247 variants were detected by EM-mosaic $(n=13)$, MosaicHunter $(n=6)$, or by both algorithms $(n=4)$. All 23 248 candidates were tested via MiSeq amplicon sequencing of blood and cardiac tissue DNAs; 15 of 23 249 unique candidate mosaics were confirmed (Table 2, S7), including a CCNC variant that was identified in 250 two different CHD tissues from proband 1-01684. Ten (86\%) confirmed mosaic variants were detected in 251 blood and cardiac tissues (MAF>0.01), four were found only in cardiac tissue, and one was found only in 252 blood. Of the 7 mosaics detected by blood WES analysis, 4 were confirmed in the corresponding cardiac 253 tissue sample. Remarkably, five confirmed cardiac tissue mosaic variants occurred in one proband (1254 07004), one of which was also present in blood DNA. These analyses indicate a frequency of coding mosaics $(0.4>\mathrm{VAF}>0.1)$ in the cardiac tissues of 0.14 per individual ( 9 of 66 probands), which approximated our estimate of 0.14 blood mosaics per individual (Fig S8A). Despite these similar frequencies, multiple distinct mosaic variants were identified in these tissues. Mosaics with highest VAF were more likely to be found in both tissues (Mann-Whitney $U$ Test $P=0.019$ ), presumably indicating that the mutation occurred earlier in lineage development (Fig S9).

\section{Table 2. Mosaics detected in individuals with matched cardiovascular tissue and blood} $<$ Insert Table 2; see end of document > Characteristics of mosaic variants predicted for individuals with blood and cardiovascular tissue WES data available. Among 15 mosaics, 5 were detected via analysis of blood WES, 8 were detected from cardiovascular tissue WES, and 2 were detected by both approaches. Six of $7(86 \%)$ mosaics detected from analysis of blood were present in both DNA sources with MiSeq VAF $\geq 0.01$. Two additional variants previously identified as de novo germline variants in blood WES were absent from CHD tissue WES. Minimum 1023 MiSeq reads used to determine VAF. Abbreviations: AD, allelic depth (reference, alternate); AO, aorta; AtrSpt, atrial septum; Bmis, benign missense; Dmis, deleterious missense; LOF, Loss of function variant; LV, left ventricle; RV, right ventricle; VAF, variant allele fraction. 
Our prior genetic studies of CHD studies showed that damaging de novo variants typically

275 occurred in genes highly expressed in the top quartile of the developing E9.5 mouse heart (HHE).\{Zaidi

276 2013; Homsy 2015\} or contributed to CHD in mouse models \{Jin 2017\}. Among the 342 mosaic variants

277 identified from blood or cardiac tissue analyses that were not false by MiSeq, 65 altered these HHE

278 and/or mouse CHD genes ( $n=4558$, Table S8). RefSeq functional annotation predicted 52 variants as

279 likely-damaging variants (LOF, Dmis), and 46 as likely benign, missense (Table S8, S9). In total, we

280 observed potentially CHD-causing mosaic mutations in 25 participants, representing $1 \%$ of the 2530 total

281 participants in our CHD cohort. Among these 25 mosaics, we confirmed 22/22 (100\%) candidates tested

282 via MiSeq. Notably, multiple likely-damaging mosaic variants altered genes (ISL1, SETD2, NOVA2,

283 SMAD9, LZTR1, KCTD10, KCTD20, FZD5, and QKI ) involved in key developmental pathways, which

284 may account for the extra-cardiac phenotypes observed in these patients (Table 3, S10). There was no

285 difference in the proportion of individuals with extracardiac features among those with damaging mosaic

286 variants compared to the overall cohort ( $11 / 25$ vs $909 / 2521, P=0.68)$, and there was a wide range of

287 CHD subtypes. Five subjects carried additional de novo LoF or Dmis variants (1-06216, TYRP1; 1-

288 04046, KRT13; 1-06677, TRIP4; 1-05011, KDM5B; 1-00018, SBF1) and 4 genes harbored de novo LoF

289 or Dmis variants other than those listed in Table 3 (FBN1; PKD1; LZTR1; PIK3C2G). No CNVs were

290 detected in these subjects, with the exception of 1-00192 (duplication at chr15:22062306-23062355; non-

291 overlapping with the GLYR1 mosaic).

292 If mosaic variants were unrelated to CHD, we would expect similar allelic fractions between

293 mosaics with variants predicted as likely damaging or likely benign. However, we found that the allele

294 fraction of likely damaging variants was significantly higher (Mann-Whitney U Test $P=0.001$, Fig 4A).

295 Moreover, among mosaic variants in genes that are not included among HHE or mouse CHD genes, we

296 found no significant difference of allele fraction ( $P=0.985$, Fig 4B). We repeated these analyses using

297 less stringent posterior odds cutoffs of 2 and 5 and found the same result (Fig S10). Together these data

298 support our conclusion that at least some likely-damaging mosaic variants identified here contribute to

299 CHD. These results were determined independently of MiSeq validation results. 


\section{Discussion}

There were 25 potentially-pathogenic mosaic mutations based on known gene function and patient phenotype. Some of these probands have previously described rare LoF/Dmis variants, though none are likely pathogenic for CHD \{Jin 2017\}. Additionally, some genes were previously found to have LoF/Dmis variants among other individuals in this CHD cohort. Abbreviations: ASD, atrial septal defect; BAV, bicuspid aortic valve; Dmis, deleterious missense; episcore, haploinsufficiency score (percentile rank) \{Han 2018\}; Heart Exp, heart expression percentile rank; LoF, loss-of-function; pLI, probability of loss-of-function intolerance \{gnomAD\}; PCGC, Pediatric Cardiac Genomics Consortium; VAF, variant allele fraction; VSD, ventricular septal defect. ${ }^{*} \mathrm{VAF}$ refers to CHD tissue WES.

reproductive implications for proband and parents. Individuals with mosaic mutations are generally clinically less severely affected for conditions that affect multiple parts of the body Happle 1986; Wallis 1990; Cohn 1990; Etheridge 2011; Donkervoort 2015; Weinstein 2016\}. Mutations that occur postzygotically should have no recurrence risk for the parents and could have a recurrence risk of less than $50 \%$ for the proband depending on gonadal involvement. This study is among the first investigations of the role of post-zygotic mosaic mutations in CHD. We developed a new computational method to robustly detect mosaic single nucleotide variants from blood WES data at standard read depth. Applying this method to a cohort of $2530 \mathrm{CHD}$ patients, we detected 309 high-confidence mosaics (with a confirmation frequency of $88 \%$ in a subset of variants assessed) or 0.12 variants per proband. Sequencing of cardiac tissue to greater depth identified an additional 8 mosaic variants that had not been detected in blood WES, 6 of which are present in cardiac tissue but not blood. We found significantly more variants per proband in cardiac tissue DNA ( 0.23 variants per proband) than in blood DNA ( 0.12 variants per proband; $p=0.02$ ). While the increased numbers of mosaic variants in cardiac tissue DNA vs blood DNA may reflect technical differences such as sequencing read depth of cardiac tissue DNA vs blood DNA, it is possible that somatic variation occurs more frequently in cardiac tissue of $\mathrm{CHD}$ probands than in their blood. Whether or not there are more cardiac tissue mosaic variants in CHD probands than blood DNA variants, we found 10 mosaic variants among $66 \mathrm{CHD}$ proband cardiac tissues with a higher VAF in tissue than in blood (Table 2) and 5 variants among these individuals with a higher VAF in blood than in tissue. In total, we observed potentially CHD-causing mosaic mutations in 25 participants, representing 
337 significantly greater alternative allele fraction than likely-benign mosaics, suggesting that some of these variants contribute to CHD. Comparison of blood and cardiovascular tissues demonstrated tissue-specific

339 mosaic variants, though those variants with a higher VAF were more likely to be shared between tissues.

340 Due to limitations of conventional clinical interpretation for both mosaic and constitutional CHD variants

341 (Supplemental Methods), we cannot know with complete certainty which among these 25 variants is

342 pathogenic and instead propose that, among our detected mosaics, the 23 detected from blood WES

343 data provide an estimate of the disease-causing mosaics detectable in blood with standard exome-

344 sequencing read depth. Nine of these variants affect genes known to have a role in cardiac development:

345 ISL1, SETD2, NOVA2, QKI, SMAD9, LZTR1, KCTD10, KCTD20, and FZD5.

The mosaic LOF mutation in ISL1 is likely to be the cause of CHD in participant 1-05095. ISL1 is

347 a transcription factor essential to normal cardiac development that regulates expression of NKX, GATA,

348 and TBX family genes \{Golzio 2012; Colombo 2018\} and controls secondary heart field differentiation and

349 atrial septation \{Colombo 2018; Briggs 2012\}. ISL1 deficiency has been shown to lead to severe CHD in

350 mice \{Cai 2003; Golzio 2012\}. Participant 1-05095 has an isolated atrial septal defect consistent with a

351 secondary heart field defect phenotype $\{$ Stevens 2010$\}$ and has no other previously reported damaging

352 germline variants in CHD-related genes.

Damaging germline de novo variants in CHD subjects are enriched in genes related to chromatin

354 modification and RNA processing \{Homsy 2015; Jin 2017\}. Three genes with damaging mosaic variants

355 discovered here have related functions. SETD2 is a histone methyltransferase required for embryonic

356 vascular remodeling $\{\mathrm{Hu} 2010\}$; it is both sensitive to haploinsufficiency and highly expressed in the heart

357 during development. NOVA2 is a key alternative-splicing regulator involved in angiogenesis that has been

358 shown to disrupt vascular lumen formation when depleted \{Giampietro 2015\}. QKI encodes an RNA-

359 binding protein that regulates splicing, RNA export from the nucleus, protein translation, and RNA stability

$360\{$ Lauriat 2008\}. QKI is also highly expressed in the heart during development and has been shown to

361 cause CHD and other blood vessel defects in mice when dysregulated \{Noveroske 2002\}.

362 Other damaging mosaic variants affect processes known to be relevant to CHD. SMAD9 is

363 involved in the TGF-beta signaling pathway. TGF-beta signaling plays a critical role in cardiac

364 development and cardiovascular physiology, leading to pulmonary arterial hypertension and cardiac 
abnormalities in mice when dysregulated \{Drake 2015; Soubrier 2013\}. LZTR1 encodes a member of the BTB-Kelch superfamily that is highly expressed in the heart during development and has been associated with Noonan \{Yamamoto 2015; Ghedira 2017\} and DiGeorge Syndromes \{Kurahashi 1995\}, both of which are characterized by CHD. KCTD10 binds to and represses the transcriptional activity of TBX5 (T-box transcription factor), which plays a dose-dependent role in the formation of cardiac chambers $\{$ Tong 2014\}. KCTD10 is highly expressed in the heart during development and has been shown to produce

371 CHD in mice when dysregulated \{Ren 2014\}. KCTD20 is a positive regulator of Akt $\{$ Nawa 2013\} also

372 highly expressed in the heart during development. FZD5 is haploinsufficient and encodes a

373 transmembrane receptor involved in Wnt, mTOR, and Hippo signaling pathways and has been shown to 374 play a role in cardiac development \{Dawson 2013\}.

Finally, two mosaic variants found in cardiac tissue, genes encoding RFX3 and PIK3C2G, may be disease-relevant. PIK3C2G is a signaling kinase involved in cell proliferation, survival, and migration, as well as oncogenic transformation and protein trafficking \{OMIM: 609001; RefSeq\}. The effects of PIK3C2G haploinsufficiency during cardiac development has not been characterized. RFX3 is a highlyconstrained ciliogenic transcription factor that leads to pronounced laterality defects $\{$ Rasmdell 2005$\}$ and disruption of RFX3 leads to congenital heart malformations in mice \{Lo 2011 MGI: 5560494\}. Notably the RFX3 LoF variant has a 4-fold higher VAF in cardiac tissue than in blood.

383 that the VAF correlates with time of mutation acquisition and disease burden \{Belickova 2016; Sallman

384 2016; Happle 1986\}. In this study, we used VAF as a proxy for cellular percentage and mutational timing,

385 with increasing VAF corresponding to events occurring earlier in development. Thus, we assume that

386 CHD-causal mosaic events identified in blood-derived DNA occurred during or shortly after the

387 gastrulation process ( $3^{\text {rd }}$ week of development) \{Moorman 2003\} in the mesodermal progenitor cells that

388 differentiate into both heart precursor cells (cardiogenic mesoderm) and blood precursor cells

389 (hemangioblasts). We found that in CHD-relevant genes, mosaic sites predicted to be damaging tended

390 to have higher VAF than sites predicted to be likely benign, consistent with the hypothesis that these

391 mutations arose early in fetal development and play significant roles in CHD. However, additional

392 functional studies are necessary to fully assess causality. . 
Finally, we recognize that while our method is able to detect a large fraction of mosaic variants in

394 blood, our calibrated estimates for the true number of mosaics suggest there are a non-negligible number

395 of additional mutations that were not identified by our method. At our current average sequencing depth

396 of $60 x$, we have limited sensitivity in the low $\operatorname{VAF}(<0.05)$ range. To reliably identify these low allelic

397 fraction sites, ultra-deep sequencing will be critical to distinguishing true variants from noise. At 500x, we

398 estimate detection sensitivity for mosaic events at VAF 0.05 to be above $80 \%$. We also recognize age-

399 related clonal hematopoiesis \{Jaiswal 2014; Genovese 2014\} as a potential confounding factor in somatic

400 mutation detection; however, our study cohort includes mostly pediatric cases and we did not observe

401 mosaic mutations in genes related to clonal expansion (e.g. ASXL1, DNMT3A, TET2, JAK2) nor did we

402 observe a relationship between proband age and mosaic rate (Fig S11, S12), suggesting minimal impact

403 from this process.

404

405 Conclusions

406 This study is among the first investigations of the role of post-zygotic mosaic mutations in CHD.

407 Despite limitations in sequencing depth and sample type, EM-mosaic was able to detect 309 high-

408 confidence mosaics with resequencing confirmation in $88 \%$ of cases assessed. Using MosaicHunter, an

409 additional 64 candidate mosaic sites were identified, of which 23/46 (50\%) candidates from blood DNA

410 and 4/6 (67\%) from CHD tissue DNA validated. In total, we observed potentially CHD-causing mosaic

411 mutations in 25 participants, representing $1 \%$ of our $\mathrm{CHD}$ cohort, and propose that these 25 cases

412 provide an estimate of the disease-causing mosaics detectable in blood with standard exome-sequencing

413 read depth. Additionally, we found that in CHD-related genes, likely-damaging mosaics have significantly

414 greater alternative allele fraction than likely benign mosaics, suggesting that many of these variants

415 cause CHD and occurred early in development. In the subset of our cohort for which cardiovascular

416 tissue samples were available, we show that mosaics detected in blood can also be found in the disease-

417 relevant tissue and that, while the VAF for mosaic variants often differed between blood and

418 cardiovascular tissue DNA, variants with higher VAF were more likely to be shared between tissues.

419 Given current limitations in sequencing depth and on the availability of relevant tissues, particularly for

420 conditions impacting internal organs like the heart, the full extent of the role of mosaicism in many 
421 diseases remains to be explored. However, as datasets containing larger numbers of blood and other

422 tissue samples sequenced at higher depths become increasingly available, we will be able to more fully

423 characterize the biological processes underlying post-zygotic mutation and, by extension, the contribution

424 of mosaicism to disease using the methods presented here.

426 Methods

427 Samples and Sequencing Data

We analyzed WES data from 2530 Congenital Heart Disease (CHD) proband-parents trio families

429 who were recruited as part of the Pediatric Cardiac Genomics Consortium (PCGC) study \{Homsy 2015;

430 Jin 2017\}. Genomic DNA from venous blood or saliva was captured using Nimblegen v.2 exome capture

431 reagent (Roche) or Nimblegen SeqCap EZ MedExome Target Enrichment Kit (Roche) followed by

432 Illumina DNA sequencing (paired-end, 2x75bp) \{Jin 2017, Zaidi 2013\}. Genomic DNA from 70 surgically-

433 discarded cardiovascular tissue samples (2-10mg) was isolated using DNeasy Blood \& Tissue Kit

434 (QIAgen), then captured using xGen Exome Research Panel v1.0 reagent (IDT) followed by Illumina DNA

435 sequencing (paired-end, 2x75bp). Sequence reads were mapped to the hg19 human reference genome

436 with BWA-MEM and BAM files were further processed following GATK Best Practices, which included

437 duplication marking, indel realignment, and base quality recalibration steps. Blood and saliva samples

438 had sample average depth 60x and cardiovascular tissue samples had sample average depth 160x.

De Novo Variant Calling and Annotation

We processed our sample BAMs and called variants on a per-trio basis using SAMtools (v1.3.1-

44242 ) and BCFtools (v1.3.1-174). Pileups were generated using samtools 'mpileup' command with mapQ

44320 and baseQ 13 to minimize the effect of poorly mapped reads on variant allele fraction, followed by

444 bcftools 'call' using a cutoff of 1.1 for the posterior probability of the homozygous reference genotype

445 parameter (-p) to capture additional sites with variant allele fraction suggestive of post-zygotic origin that

446 would otherwise be excluded under the default threshold of 0.01. To identify de novo mutations from trio

447 VCF files, we selected sites with (i) a minimum of 6 reads supporting the alternate allele in the proband

448 and (ii) for both parents, a minimum depth of 10 reads and 0 alternate allele read support. Variants were 
449 then annotated using ANNOVAR (v2017-07-17) to include information from refGene, gnomAD (March

450 2017), 1000 Genomes (August 2015), ExAC, genomicSuperDups, CADD (v1.3) COSMIC (v70), and

$451 \mathrm{dbSNP}$ (v147) databases, as well as pathogenicity predictions from a variety of established methods

452 included as part of the dbNSFP (v3.0a) database or generated in-house (MCAP, REVEL, MVP, MPC).

453 We used REVEL \{lonnidis 2016\} to evaluate missense variant functional consequence, using the

454 recommended threshold of 0.5 corresponding to sensitivity of 0.754 and specificity of 0.891 . We used

455 spliceAl \{Jaganathan 2019$\}$ to predict the variant functional impact on splicing using the delta score

456 thresholds of 0.2 for likely pathogenic (high recall), 0.5 for pathogenic (recommended), and 0.8 for

457 pathogenic (high precision). We considered sites predicted to be Likely Gene-Disrupting (LOF) (stopgain,

458 stoploss, frameshift indels, splice-site), Deleterious Missense (Dmis; nonsynonymous SNV with

459 REVEL>0.5), or splice-damaging (Benign Missense or synonymous SNV with delta score $>0.5$ ) to be

460 damaging and likely disease causing. We considered sites predicted to be Synonymous (delta score $\leq$

4610.5 ) or Benign missense (Bmis; nonsynonymous SNV with REVEL $\leq 0.5$ and delta score $\leq 0.5$ ) to be non-

462 damaging.

Pre-processing and QC

To reduce the number of low VAF technical artifacts introduced by our variant calling approach,

466 we pre-processed our variants using a variety of filters. We first excluded indels from further analysis, as

467 their downstream model parameter estimates were less stable than those of SNVs. We then filtered our

468 variant call set for rare heterozygous coding mutations (Minor Allele Frequency (MAF) $\leq 10^{-4}$ across all

469 populations represented in gnomAD and ExAC databases). To account for regions in the reference

470 genome that are likely to affect read-depth estimates, we removed variant sites found in regions of non-

471 unique mappability (score $<1 ; 300 \mathrm{bp}$ ), likely segmental duplication (score $>0.95$ ), and known low-

472 complexity $\{$ Li 2014\}. We then excluded sites located in MUC and HLA genes and imposed a maximum

473 variant read depth threshold of 500 . We used SAMtools PV4 to exclude sites with evidence of technical

474 issues using a cutoff of 1e-3 for baseQ Bias and Tail Distance Bias and a cutoff of 1e-6 for mapQ Bias.

475 To account for potential strand bias, we used an in-house script to flag sites that have either (1) 0

476 alternate allele read support on either the forward or reverse strand or (2) $p<1 \mathrm{e}-3$ and (Odds Ratio 
$477(\mathrm{OR})<0.33$ or $\mathrm{OR}>3)$ when applying a two-sided Fisher's Exact Test to compare proportions of reference

478 and alternate allele read counts on the forward and reverse strands. We also excluded sites with cohort

479 frequency $>1 \%$, as well as sites belonging to outlier samples (with abnormally high de novo SNV (dnSNV)

480 counts, cutoff $=8$ ) and variant clusters (defined as sites with neighboring SNVs within 10bp). Finally, we

481 applied an FDR-based minimum $\mathrm{N}_{\text {alt }}$ filtering step (Fig S7) to control for false positives caused purely by

482 sequencing errors.

IGV Visualization of Low Allele Fraction de novo SNVs

To reduce the impact of technical artifacts on model parameter estimation, we manually inspected de novo SNVs with VAF<0.3 $(\mathrm{n}=558)$ using Integrative Genomics Viewer (v2.3.97) to visualize the local read pileup at each variant across all members of a given trio family. We focused on the allele

488 fraction range 0.0-0.3 since this range is enriched for technical artifacts that could potentially impact downstream parameter estimation. Sites were filtered out if (1) there are inconsistent mismatches in the reads supporting the mosaic allele, (2) the site overlaps or is adjacent to an indel, (3) the site has low MAPQ or is not Primary alignment, (4) there is evidence of technical bias (strand, read position, tail distance), or (5) the site is mainly supported by soft-clipped reads.

\section{Expectation-Maximization to Estimate Prior Mosaic Fraction and Control FDR}

Current estimates for the fraction of de novo events occurring post-zygotically are unstable due to

496 differences in study factors such as variant calling methods, average sequencing depth, and paternal

497 ages. In order to use this fraction as a prior probability in our posterior odds and false discovery

498 calculations, we reason that this value must be estimated from the data itself. We used an expectation-

499 maximization algorithm to jointly estimate the fraction of mosaics among apparent de novo mutations and

500 to calculate a per-site likelihood ratio score. This initial mosaic fraction estimate gives us a prior

501 probability of mosaicism, independent of sequencing depth or variant caller, and allows us to calculate for

502 each variant the posterior odds that a given site is mosaic rather than germline. To control for false

503 discovery among our predicted mosaic candidates, we chose a posterior odds threshold of 10 to restrict

504 FDR to $9.1 \%$. 


\section{Mosaic Mutation Detection Model}

To distinguish variant sites that show evidence of mosaicism from germline heterozygous sites,

508 we modeled the number of reads supporting the variant allele $\left(N_{\mathrm{alt}}\right)$ as a function of the total site depth

$509(M)$. In the typical case, $N_{\text {alt }}$ follows a binomial model with parameters $N=$ site depth and $p=$ mean VAF.

510 However, we observed notable overdispersion in the distribution of variant allele fraction compared to the

511 expectations under this binomial model (Fig S6). To account for this overdispersion, we instead modeled

$512 \quad N_{a l t}$ using a beta-binomial distribution \{Heinrich 2012; Ramu 2013\}. We estimated an overdispersion

513 parameter $\theta$ for our model as follows: for site depth values $N$ in the range 1 to 500 , we (1) bin variants by

514 identifying all sites with depth $N$, (2) calculate a maximum-likelihood estimate $\theta$ value using $N$ and all $N_{\text {alt }}$

515 values for variants in a given bin, and (3) estimate a global $\theta$ value by taking the average of $\theta$ values

516 across all bins, weighted by the number of variants in each bin. We then used $\theta$ in our expectation-

517 maximization approach to jointly estimate prior mosaic fraction and to calculate per-site likelihood ratios.

518 To calculate the posterior odds that a given variant arose post-zygotically, we first calculated a

519 likelihood ratio (LR) of two models: $\mathrm{M}_{0}$ : germline heterozygous variant, and $\mathrm{M}_{1}$ : mosaic variant. Under our

520 null model $\mathrm{M}_{0}$, we calculated the probability of observing $\mathrm{N}_{\text {alt }}$ from a beta-binomial distribution with site

521 depth $N$, observed mean germline VAF $p$, and overdispersion parameter $\theta$. Under our alternate model $\mathrm{M}_{1}$,

522 we calculated the probability of observing $N_{\text {alt }}$ from a beta-binomial distribution with site depth $N$,

523 observed site VAF $p=N_{\text {alt }} / N$, and overdispersion parameter $\theta$. Finally, for each variant, we calculated LR

524 by using the ratio of probabilities under each model and posterior odds by multiplying LR by our E-M

525 estimated prior mosaic fraction estimate. We defined mosaic sites as those with posterior odds greater

526 than 10 (corresponding to $9.1 \%$ FDR). We used posterior odds in this context to be able to control for

527 false discovery, but we output similarly valid p-value and likelihood ratio scores for each de novo SNV.

Mutation Confirmation by MiSeq Amplicon Sequencing

530 Chromosome coordinates were expanded $500 \mathrm{bp}$ upstream and downstream of the candidate

531 mosaic variants in the UCSC Genome Browser. Primer 3 Plus software was used to design forward and

532 reverse primers to generate $150-300$ bp amplimers containing the candidate site. PCR reactions 
533 consisting of genomic DNA, primers, and Phusion polymerase were amplified by thermal cycling and

534 purified with AMPure XP beads. The purified PCR product was quantified, and 0.5-1.0 ng of product was

535 used to construct Nextera XT libraries according to the protocol published by Illumina. Libraries were

536 purified using AMPure XP beads, and final libraries were quantified and pooled to undergo sequencing

537 through Illumina MiSeq.

538 We experimentally tested for the presence our predicted post-zygotic sites in the original blood

539 DNA and cardiovascular tissue DNA samples using Illumina MiSeq Amplicon sequencing. The Amplicon

540 Deep Sequencing workflow, optimized for the detection of somatic mutations in tumor samples, offers

541 ultra-high sequencing depth (>1000x) that gives us the resolution to confirm low VAF variants, accurately

542 estimate site VAF, and to distinguish true variant calls from technical artifacts. Mosaic candidates were

543 considered validated if the variant allele matched the MiSeq call and both the mosaic VAF and MiSeq

544 VAF indicated post-zygotic origin $(\mathrm{VAF}<0.45)$.

545 Mosaic candidates were selected for confirmation on the basis of VAF, plausible involvement in

546 CHD (based on predicted pathogenicity and HHE status), and detection method (Table S11; Table S12).

547 We sampled mosaics from both ends of the VAF spectrum to evaluate our ability to distinguish high VAF

548 mosaics $(\mathrm{VAF}>0.2 ; \mathrm{n}=29)$ from germline variants and to distinguish low $\mathrm{VAF}$ mosaics $(\mathrm{VAF}<=0.1 ; \mathrm{n}=52)$

549 from technical artifacts. Confirmation rate across different VAF bins is shown in Figure S13. We also

550 selected for confirmation mosaics detected uniquely by either EM-mosaic or MosaicHunter, for the sake

551 of method comparison (Table 1).

552 To examine a potential source of bias in our candidate selection process, we compared the

553 posterior odds distribution of selected candidate mosaics $(n=97)$ against those not chosen $(n=212)$. We

554 found that our tested candidates had lower posterior odds than untested mosaics $\left(\right.$ mean $_{\text {tested }}=5.382$,

555 mean $_{\text {untested }}=7.050, \log _{10}$-scale; Mann Whitney $\cup P=0.002$ ) (Fig 14), suggesting that our validation rate is

556 not buoyed by testing variants with the strongest evidence of mosaicism. For method development

557 purposes, we intentionally focused on mosaics with lower posterior odds as these fall in the VAF range

558 for which it is most difficult to distinguish germline from mosaic.

560 Investigating the relationship between VAF and pathogenicity 
We hypothesized that mosaic contribution to disease is positively correlated with cellular percentage and by extension mutational timing. Here, we used variant allele fraction as a proxy for

563 cellular percentage. We grouped mosaics into likely-damaging and likely-benign and compared the

564 distribution of allele fraction in CHD-related genes. We defined likely-damaging variants as: (a) likely 565 gene-disrupting (LOF) variants (including premature stop-gain, frameshifting, and variants located in 566 canonical splice sites), (b) missense variants predicted to be damaging by REVEL \{loannidis 2016\} (with 567 score $\geq 0.5$ ) or (c) missense variants and synonymous predicted to be splice-damaging by spliceAl (with 568 score $>0.5)$. One of the main findings from previous CHD studies is that damaging de novo variants in

569 genes highly expressed in the developing heart ("HHE", ranked in the top $25 \%$ by cardiac expression data 570 in mouse at E14.5 \{Zaidi 2013; Homsy 2015\}) contribute to non-isolated CHD cases that have additional 571 congenital anomalies or neurodevelopmental disorders. Therefore, we considered the union of HHE 572 genes and known candidate CHD genes \{Jin 2017\} as CHD-related genes ( $n=4558)$. For mosaics in 573 CHD-related genes and for mosaics in other genes, we used a Mann-Whitney U Test to compare the VAF 574 distributions of likely-damaging and likely-benign groups.

\section{Estimated contribution of mosaicism to $C H D$}

We identified likely disease-causing mosaic mutations on the basis of predicted pathogenicity and 578 presence in genes involved in biological processes relevant to CHD or developmental disorders. Each 579 mosaic mutation was annotated with gene-specific information, including heart expression percentile, 580 probability of loss-of-function intolerance (pLI) score $\{$ Lek 2016\}, whether dysregulation causes CHD in 581 mice \{Smith 2018; Finger 2017\}, and gene function \{NCBI RefSeq\}. We focused on HHE genes, genes

582 with high $\mathrm{pLI}(\mathrm{pLI}>0.9)$, genes that cause CHD phenotypes in mice, and genes involved in key

583 developmental processes such as Wnt, mTOR, and TGF-beta signaling pathways. Then, for each patient,

584 we used the clinical phenotype to further prioritize mosaic mutations most likely contributing to that 585 individual's clinical features. Detailed mutation annotation and clinical phenotypes for the mosaic carriers 586 described above can be found in Table S10. We estimate the contribution of mosaicism to CHD as the 587 percentage of individuals carrying likely disease-causing mosaic mutations among all individuals in our 588 CHD cohort. 
592 Abbreviations

593 ASD: Autism Spectrum Disorder

594 CHD: Congenital Heart Disease

595 dnSNV: de novo SNV

596 Dmis: Deleterious Missense mutation

$597 \quad \mathrm{DP}_{\text {site }}$ : Total Read Depth at a variant site

598 DP $_{\text {sample }}$ : Sample-wide Average Read Depth

599 ExAC: Exome Aggregation Consortium

600 FDR: False Discovery Rate

601 gnomAD: Genome Aggregation Database

602 HHE: High Heart Expression

603 LOF: Loss-of-Function

604 LR: Likelihood Ratio

605 MAF: Minor Allele Fraction

$606 \quad \mathrm{~N}:$ Total Read Depth

$607 \quad \mathrm{~N}_{\text {alt }}$ : Alternate Allele Read Depth

608 OR: Odds Ratio

609 PCGC: Pediatric Cardiac Genomics Consortium

610 pLI: Probability of Loss-of-Function Intolerance

611 PV4: P-value for strand bias, baseQ bias, mapQ bias and tail distance bias (SAMtools)

612 SNV: Single Nucleotide Variant

613 VAF: Variant Allele Fraction

614 WES: Whole Exome Sequencing 


\section{References}

Acuna-Hidalgo, R., Bo, T., Kwint, M. P., van de Vorst, M., Pinelli, M., Veltman, J. A., ... Gilissen, C. (2015). Post-zygotic Point Mutations Are an Underrecognized Source of De Novo Genomic Variation. The American Journal of Human Genetics, 97(1), 67-74. http://doi.org/10.1016/J.AJHG.2015.05.008

Belickova, M., Vesela, J., Jonasova, A., Pejsova, B., Votavova, H., Merkerova, M. D., ... Cermak, J. (2016). TP53 mutation variant allele frequency is a potential predictor for clinical outcome of patients with lower-risk myelodysplastic syndromes. Oncotarget, 7(24), 36266-36279. http://doi.org/10.18632/oncotarget.9200

Biesecker, L. G., \& Spinner, N. B. (2013). A genomic view of mosaicism and human disease. Nature Reviews Genetics, 14(5), 307-320. http://doi.org/10.1038/nrg3424 septal defects with special emphasis on the role of the dorsal mesenchymal protrusion. Differentiation, 84(1), 117-130. http://doi.org/10.1016/j.diff.2012.05.006 cardiac progenitor population that proliferates prior to differentiation and contributes a majority of cells to the heart. Developmental Cell, 5(6), 877-89. Retrieved from http://www.ncbi.nlm.nih.gov/pubmed/14667410 (2013). Sensitive detection of somatic point mutations in impure and heterogeneous cancer samples. Nature Biotechnology, 31(3), 213-219. http://doi.org/10.1038/nbt.2514 imperfecta due to parental mosaicism for a dominant mutation in a human type I collagen gene (COL1A1). American Journal of Human Genetics, 46(3), 591-601. Retrieved from http://www.ncbi.nlm.nih.gov/pubmed/2309707 Colombo, S., de Sena-Tomás, C., George, V., Werdich, A. A., Kapur, S., MacRae, C. A., \& Targoff, K. L. (2017). nkx genes establish SHF cardiomyocyte progenitors at the arterial pole and 
pattern the venous pole through Isl1 repression. Development, dev.161497. http://doi.org/10.1242/dev.161497

647 Dawson, K., Aflaki, M., \& Nattel, S. (2013). Role of the Wnt-Frizzled system in cardiac pathophysiology: A rapidly developing, poorly understood area with enormous potential. Journal of Physiology, 591(6), 1409-1432. http://doi.org/10.1113/jphysiol.2012.235382 (2011). A framework for variation discovery and genotyping using next-generation DNA sequencing data. Nature Genetics, 43(5), 491-8. http://doi.org/10.1038/ng.806

Donkervoort, S., Hu, Y., Stojkovic, T., Voermans, N. C., Foley, A. R., Leach, M. E., ... Bönnemann, C. G. (2015). Mosaicism for Dominant Collagen 6 Mutations as a Cause for Intrafamilial Phenotypic Variability. Human Mutation, 36(1), 48-56. http://doi.org/10.1002/humu.22691 nucleotide mosaicisms contribute to the etiology of autism spectrum disorder and autistic traits and the origin of mutations. Human Mutation, 38(8), 1002-1013. http://doi.org/10.1002/humu.23255

660 Drake, K. M., Comhair, S. A., Erzurum, S. C., Tuder, R. M., \& Aldred, M. A. (2015). Endothelial Chromosome 13 Deletion in Congenital Heart Disease-associated Pulmonary Arterial Hypertension Dysregulates SMAD9 Signaling. American Journal of Respiratory and Critical Care Medicine, 191(7), 850-854. Firouzi, M. (2011). Somatic mosaicism contributes to phenotypic variation in Timothy syndrome. American Journal of Medical Genetics Part A, 155(10), 2578-2583. 
Fischbach, G. D., \& Lord, C. (2010). The Simons Simplex Collection: A Resource for Identification of Autism Genetic Risk Factors. Neuron, 68(2), 192-195.

673 http://doi.org/10.1016/j.neuron.2010.10.006 (2015). Genome-wide patterns and properties of de novo mutations in humans. Nature Genetics, 47(7), 822-826. https://doi.org/10.1038/ng.3292

Freed, D., \& Pevsner, J. (2016). The Contribution of Mosaic Variants to Autism Spectrum Disorder. PLOS Genetics, 12(9), e1006245. http://doi.org/10.1371/journal.pgen.1006245

Fryxell, K. J., \& Moon, W.-J. (2005). CpG Mutation Rates in the Human Genome Are Highly Dependent on Local GC Content. Molecular Biology and Evolution, 22(3), 650-658. https://doi.org/10.1093/molbev/msi043 McCarroll, S. A. (2014). Clonal Hematopoiesis and Blood-Cancer Risk Inferred from Blood DNA Sequence. New England Journal of Medicine, 371(26), 2477-2487. http://doi.org/10.1056/NEJMoa1409405

Ghedira, N., Kraoua, L., Lagarde, A., Abdelaziz, R. Ben, Olschwang, S., Desvignes, J. P., ... Mrad, R. (2017). Further Evidence for the Implication of LZTR1, a Gene not Associated with the RasMapk Pathway, in the Pathogenesis of Noonan Syndrome. Biology and Medicine, 09(06), 4-7. http://doi.org/10.4172/0974-8369.1000414 (2015). The alternative splicing factor Nova2 regulates vascular development and lumen formation. Nature Communications, 6, 1-15. http://doi.org/10.1038/ncomms9479 Golzio, C., Havis, E., Daubas, P., Nuel, G., Babarit, C., Munnich, A., ... Etchevers, H. C. (2012). ISL1 Directly Regulates FGF10 Transcription during Human Cardiac Outflow Formation. PLoS ONE, 7(1), e30677. http://doi.org/10.1371/journal.pone.0030677 Happle, R. (1986). The McCune-Albright syndrome: a lethal gene surviving by mosaicism. Clinical Genetics, 29(4), 321-4. Retrieved from http://www.ncbi.nlm.nih.gov/pubmed/3720010 
Happle, R. (1993). Mosaicism in human skin. Understanding the patterns and mechanisms. Archives of Dermatology, 129(11), 1460-70. Retrieved from http://www.ncbi.nlm.nih.gov/pubmed/8239703 Heinrich, V., Stange, J., Dickhaus, T., Imkeller, P., Krüger, U., Bauer, S., ... Krawitz, P. M. (2012). The allele distribution in next-generation sequencing data sets is accurately described as the result of a stochastic branching process. Nucleic Acids Research, 40(6), 2426-2431. http://doi.org/10.1093/nar/gkr1073

Homsy, J., Zaidi, S., Shen, Y., Ware, J. S., Samocha, K. E., Karczewski, K. J., ... Chung, W. K. (2015). De novo mutations in congenital heart disease with neurodevelopmental and other congenital anomalies. Science, 350(6265), 1262-1266. http://doi.org/10.1126/science.aac9396 Hu, M., Sun, X.-J., Zhang, Y.-L., Kuang, Y., Hu, C.-Q., Wu, W.-L., ... Chen, Z. (2010). Histone H3 lysine 36 methyltransferase Hypb/Setd2 is required for embryonic vascular remodeling. Proceedings of the National Academy of Sciences, 107(7), 2956-2961. http://doi.org/10.1073/pnas.0915033107 Huang, A. Y., Zhang, Z., Ye, A. Y., Dou, Y., Yan, L., Yang, X., .. Wei, L. (2017). MosaicHunter: accurate detection of postzygotic single-nucleotide mosaicism through next-generation sequencing of unpaired, trio, and paired samples. Nucleic Acids Research, 45(10), e76-e76. http://doi.org/10.1093/nar/gkx024 loannidis, N. M., Rothstein, J. H., Pejaver, V., Middha, S., McDonnell, S. K., Baheti, S., ... Sieh, W. (2016). REVEL: An Ensemble Method for Predicting the Pathogenicity of Rare Missense Variants. The American Journal of Human Genetics, 99(4), 877-885. http://doi.org/10.1016/j.ajhg.2016.08.016 Jaiswal, S., Fontanillas, P., Flannick, J., Manning, A., Grauman, P. V., Mar, B. G., ... Ebert, B. L. (2014). Age-Related Clonal Hematopoiesis Associated with Adverse Outcomes. New England Journal of Medicine, 371(26), 2488-2498. http://doi.org/10.1056/NEJMoa1408617 Jamuar, S. S., Lam, A.-T. N., Kircher, M., D'Gama, A. M., Wang, J., Barry, B. J., ... Walsh, C. A. (2014). Somatic Mutations in Cerebral Cortical Malformations. New England Journal of Medicine, 371(8), 733-743. http://doi.org/10.1056/NEJMoa1314432 
Jin, S. C., Homsy, J., Zaidi, S., Lu, Q., Morton, S., DePalma, S. R., ... Brueckner, M. (2017).

Contribution of rare inherited and de novo variants in 2,871 congenital heart disease probands. Nature Genetics, 49(11), 1593-1601. http://doi.org/10.1038/ng.3970

Krupp, D. R., Barnard, R. A., Duffourd, Y., Evans, S. A., Mulqueen, R. M., Bernier, R., ... O’Roak, B. American Journal of Human Genetics, 101(3), 369-390. http://doi.org/10.1016/j.ajhg.2017.07.016

Kurahashi, H., Akagi, K., Inazawa, J., Ohta, T., Niikawa, N., Kayatani, F., ... Nishisho, I. (1995). Isolation and characterization of a novel gene deleted in DiGeorge syndrome. Human Molecular Genetics, 4(4), 541-9. Retrieved from http://www.ncbi.nlm.nih.gov/pubmed/7633402 American Journal of Human Genetics, 90(6), 1108-1115. http://doi.org/10.1016/j.ajhg.2012.05.006 (2008). Developmental expression profile ofquaking, a candidate gene for schizophrenia, and its target genes in human prefrontal cortex and hippocampus shows regional specificity. Journal of Neuroscience Research, 86(4), 785-796. http://doi.org/10.1002/jnr.21534

Lee, J. H., Huynh, M., Silhavy, J. L., Kim, S., Dixon-Salazar, T., Heiberg, A., ... Gleeson, J. G. (2012). De novo somatic mutations in components of the PI3K-AKT3-mTOR pathway cause hemimegalencephaly. Nature Genetics, 44(8), 941-945. http://doi.org/10.1038/ng.2329 A. (2016). Analysis of protein-coding genetic variation in 60,706 humans. Nature, 536(7616), 285-291. http://doi.org/10.1038/nature19057

750 Li, H., Handsaker, B., Wysoker, A., Fennell, T., Ruan, J., Homer, N., ... 1000 Genome Project Data 751 Processing Subgroup. (2009). The Sequence Alignment/Map format and SAMtools. Bioinformatics, 25(16), 2078-2079. http://doi.org/10.1093/bioinformatics/btp352 

Rates, distribution and implications of postzygotic mosaic mutations in autism spectrum disorder. Nature Neuroscience, 20(9), 1217-1224. http://doi.org/10.1038/nn.4598 Manheimer, K. B., Richter, F., Edelmann, L. J., D’Souza, S. L., Shi, L., Shen, Y., ... Gelb, B. D. (2018). Robust identification of mosaic variants in congenital heart disease. Human Genetics, 137(2), 183-193. http://doi.org/10.1007/s00439-018-1871-6 (2010). The Genome Analysis Toolkit: a MapReduce framework for analyzing next-generation DNA sequencing data. Genome Research, 20(9), 1297-303. http://doi.org/10.1101/gr.107524.110

Moorman, A., Webb, S., Brown, N. A., Lamers, W., \& Anderson, R. H. (2003). Development of the heart: (1) formation of the cardiac chambers and arterial trunks. Heart (British Cardiac Society), 89(7), 806-14. Retrieved from http://www.ncbi.nlm.nih.gov/pubmed/12807866

Nawa, M., \& Matsuoka, M. (2013). KCTD20, a relative of BTBD10, is a positive regulator of Akt. BMC Biochemistry, 14(1), 27. http://doi.org/10.1186/1471-2091-14-27

Noveroske, J. K., Lai, L., Gaussin, V., Northrop, J. L., Nakamura, H., Hirschi, K. K., \& Justice, M. J. (2002). Quaking is essential for blood vessel development. Genesis (New York, N.Y. $\square: 2000$ ), 32(3), 218-30. Retrieved from http://www.ncbi.nlm.nih.gov/pubmed/11892011 (2012). Somatic Activation of AKT3 Causes Hemispheric Developmental Brain Malformations. Neuron, 74(1), 41-48. http://doi.org/10.1016/j.neuron.2012.03.010

Ramsdell, A. F. (2005). Left-right asymmetry and congenital cardiac defects: Getting to the heart of the matter in vertebrate left-right axis determination. Developmental Biology, 288(1), 1-20. http://doi.org/10.1016/J.YDBIO.2005.07.038

Ramu, A., Noordam, M. J., Schwartz, R. S., Wuster, A., Hurles, M. E., Cartwright, R. A., \& Conrad, D. F. (2013). DeNovoGear: de novo indel and point mutation discovery and phasing. Nature Methods, 10(10), 985-987. http://doi.org/10.1038/nmeth.2611 
Ren, K., Yuan, J., Yang, M., Gao, X., Ding, X., Zhou, J., ... Zhang, J. (2014). KCTD10 Is Involved in the Cardiovascular System and Notch Signaling during Early Embryonic Development. PLoS ONE, 9(11), e112275. http://doi.org/10.1371/journal.pone.0112275

Rivière, J.-B., Mirzaa, G. M., O’Roak, B. J., Beddaoui, M., Alcantara, D., Conway, R. L., ... Dobyns, W. B. (2012). De novo germline and postzygotic mutations in AKT3, PIK3R2 and PIK3CA cause a spectrum of related megalencephaly syndromes. Nature Genetics, 44(8), 934-940. http://doi.org/10.1038/ng.2331

Sallman, D. A., Komrokji, R., Vaupel, C., Cluzeau, T., Geyer, S. M., McGraw, K. L., ... Padron, E. (2016). Impact of TP53 mutation variant allele frequency on phenotype and outcomes in myelodysplastic syndromes. Leukemia, 30(3), 666-673. http://doi.org/10.1038/leu.2015.304 for next generation sequencing. Genetic Epidemiology, 35(4), n/a-n/a. http://doi.org/10.1002/gepi.20575

Smith, C. L., Blake, J. A., Kadin, J. A., Richardson, J. E., Bult, C. J., \& Mouse Genome Database Group. (2018). Mouse Genome Database (MGD)-2018: knowledgebase for the laboratory mouse. Nucleic Acids Research, 46(D1), D836-D842. http://doi.org/10.1093/nar/gkx1006 somatic variant identification from unpaired tissue samples. Bioinformatics, 32(6), 808-813. http://doi.org/10.1093/bioinformatics/btv685 Genetics and Genomics of Pulmonary Arterial Hypertension. Journal of the American College of Cardiology, 62(25), D13-D21. http://doi.org/10.1016/J.JACC.2013.10.035 Gruber, P. J. (2010). Common Variation in ISL1 Confers Genetic Susceptibility for Human Congenital Heart Disease. PLOS ONE, 5(5), e10855. http://doi.org/10.1371/journal.pone.0010855 
epilepsy-related neurodevelopmental disorders. Genetics in Medicine, 20(4), 403-410. http://doi.org/10.1038/gim.2017.114

Sun, J. X., He, Y., Sanford, E., Montesion, M., Frampton, G. M., Vignot, S., .. Yelensky, R. (2018). A computational approach to distinguish somatic vs. germline origin of genomic alterations from deep sequencing of cancer specimens without a matched normal. PLOS Computational Biology, 14(2), e1005965. http://doi.org/10.1371/journal.pcbi.1005965 morphogenesis by repressing the transcriptional activity of Tbx5a in zebrafish. Nature Communications, 5, 1-10. http://doi.org/10.1038/ncomms4153 imperfecta in a nuclear family is explained by somatic mosaicism for a lethal point mutation in the alpha $1(\mathrm{I})$ gene (COL1A1) of type I collagen in a parent. American Journal of Human Genetics, 46(6), 1034-40. Retrieved from http://www.ncbi.nlm.nih.gov/pubmed/2339700 H. (2016). Somatic mosaicism for a lethal TRPV4 mutation results in non-lethal metatropic dysplasia. American Journal of Medical Genetics Part A, 170(12), 3298-3302. http://doi.org/10.1002/ajmg.a.37942 Genetics, 52(6), 413-421. http://doi.org/10.1136/jmedgenet-2015-103018 Research, 120(6), 923-940. http://doi.org/10.1161/CIRCRESAHA.116.309140

Zaidi, S., Choi, M., Wakimoto, H., Ma, L., Jiang, J., Overton, J. D., ... Lifton, R. P. (2013). De novo mutations in histone-modifying genes in congenital heart disease. Nature, 498(7453), 220223. http://doi.org/10.1038/nature12141 
Fig 1. Mosaic detection by Expectation-Maximization. (A) Expectation-Maximization (EM) Estimation

838 to decompose the variant allele fraction (VAF) distribution of our input variants into mosaic and germline

839 distributions. The EM-estimated prior mosaic fraction was $12.15 \%$ and the mean of the mosaic VAF

840 distribution was 0.15. (B) Read depth vs. VAF distrubution of individual variants. The blue line denotes

841 mean VAF (0.49) and the red lines denote the 95\% confidence interval under our Beta-Binomial model.

842 Mosaic variants are defined as sites with posterior odds $>10$, corresponding to a False Discovery Rate of

$8439.1 \%$.Germline variants are represented in black and mosaic variants are represented in red. (C)

844 Estimated mosaic detection power as a function of average sample depth for values between 40x and

$845500 x$

Fig 2. Mutation spectrum of detected germline and mosaic variants. Rates of specific mutations were compared in germline (A), blood mosaic (B), and CHD tissue mosaic (C) variants. Transitions predominated in both variant sets.

Fig 3. Validated mosaics detected in probands with matched blood and cardiovascular tissue samples available. Validation VAF from blood compared to validation VAF from cardiovascular tissue demonstrated tissue-specific mosaicism (red) as well as shared mosaicism (blue). Predicted effect of

854 mosaic variants corresponds to marker shape.

Fig 4. Damaging mosaics in CHD-related genes have higher variant allele fraction than likely-

857 benign mosaics. (A) Among the 76 mosaics in CHD-related genes, likely damaging variants have a

858 higher VAF than likely benign (Mann-Whitney $U p=0.001$ ). (B) Among the 233 mosaics in Other (non-

859 CHD-related) genes, there is no difference in VAF based on predicted effect $(p=0.985)$. 
Union with Validated de novo SNVs from Jin et al. Nature Genetics 2017

866 CHD probands - including 2530 parent-offspring trios used in this study - to investigate the contribution

867 of rare inherited and de novo variants to CHD. They called a total of 2992 proband de novo variants,

868 including 2872 SNVs and 118 indels, and Sanger confirmed a subset of the most likely-disease causing

869 variants. Since we processed the same proband-parent trios using different variant calling pipelines, we

870 combined the results of our two approaches to provide a more complete input de novo call set for mosaic

871 variant detection.

872 We first processed our SAMtools de novo calls using our upstream filters ( $n=2396$ sites passing

873 all filters). We then applied the same upstream filters to the published dnSNVs from Jin et al. ( $n=2650$

874 sites passing all filters) before finally taking the union of these two call sets ( $n=3192)$. There were 1814

875 sites in the intersection, with 836 sites unique to the Jin et al. calls and 542 sites unique to our SAMtools

876 calls. After preprocessing, outlier removal, and FDR-based minimum $N_{\text {alt }}$ filtering, the remaining 2971

877 dnSNVs were used as input to our mosaic detection model.

\section{Mutation Spectrum Analysis}

We compared the mutation spectrum - the frequencies of all possible base changes - of our predicted mosaic candidates against the spectrum of our predicted germline heterozygous variants.

882 Under the assumption that that post-zygotic events occur randomly (i.e. due to errors in DNA replication

883 rather than a specific biological process), the mosaic mutation spectrum should not differ significantly

884 from the germline mutation spectrum. We used Pearson's Chi-square Test to test for a difference in

885 frequencies across all base changes between our predicted sets of variants. We interpreted large

886 qualitative differences in base change frequencies as evidence of technical artifacts and rejection of the

887 Chi-square null as evidence of systemic issues in our pipeline.

\section{Mosaic Detection Power Given Sample Average Coverage}

890 To model statistical power in the context of mosaic variant detection, we considered two 891 conditional probabilities: (i) the probability of detecting a mosaic event (i.e. the probability of a variant's 
892 posterior odds exceeding a threshold) given site depth $D P_{\text {site, }}$ VAF, and overdispersion parameter $\theta$ and

893 (ii) the probability of observing site depth $D P_{\text {site, }}$ given sample-wide average coverage $D P_{\text {sample. }}$

894 (i) $\operatorname{Pr}\left(\right.$ detect mosaic $\mid D P_{\text {site }}, \mathrm{VAF}, \theta$ ) was calculated by first identifying the VAF range (and by extension,

895 the range of $N_{\text {alt }}$ ) over which posterior odds > cutoff, then by integrating the beta-binomial probability

896 mass function over this range, with considerations for the probability of strand bias $\left(\mathrm{P}\left(\right.\right.$ strand bias $\left.\mid D P_{\text {site }}\right)$

$\left.897 \sim \operatorname{Binomial}\left(N_{\text {alt }}, D P_{\text {site }}, p=0.5\right)\right)$.

898 (ii) $\operatorname{Pr}\left(D P_{\text {site }} \mid D P_{\text {sample }}\right)$ follows an overdispersed poisson distribution that we approximated using a 899 negative binomial model with overdispersion parameter $\theta$ \{Sampson 2011\}. For each $D P_{\text {sample }}$ value, we

900 calculated a vector of weights corresponding to $\operatorname{Pr}\left(D P_{\text {site }} \mid D P_{\text {sample }}\right)$ for $D P_{\text {site }}$ values in the range (1, 901 1500).

Finally, we took the sum of the detection probabilities described in (i) multiplied by the weights

903 described in (ii) to determine the probability of detecting a mosaic variant given a sample average

904 coverage value $-\operatorname{Pr}\left(\right.$ detect mosaic $\left.\mid D P_{\text {sample }}\right)$. Our estimated detection power curves for a range of

905 sample average coverage values typical of exome-sequencing studies are shown in (Figure 4A). Our

906 CHD cohort was sequenced to sample average depth of $60 x$, with prior mosaic fraction $=0.121$ and

907 estimated $\theta=116$.

908 To estimate the true rate of mosaicism per exome given sample average coverage, we first split

909 our set of predicted mosaics into VAF bins of size 0.05 . For each bin above VAF 0.1, we multiplied the

910 number of mosaics by the inverse of the detection power for that given VAF bin to estimate the true count

911 of mosaic variants in that VAF range, assuming full detection power. Since EM-mosaic is underpowered

912 to detect mosaics with VAF $<0.1$ in the blood and since this range is enriched for technical artifacts that

913 potentially affect our counts, we did not apply this scaling procedure to these bins to avoid over-inflating

914 our adjusted mosaic rate estimate (Figure 4B).

\section{Filtering of MosaicHunter Candidate Variants (Fig S3)}

917 MosaicHunter was used to identify candidate mosaic variants from blood exome-sequencing trio 918 data using default settings \{Huang 2014\}. Filtering of original MosaicHunter candidate variants excluded, 919 in order, any variant present in ExAC (46634), G to T mutations with fewer than $\mathrm{N}_{\text {alt }}<10$ oxidative 
920 indicating DNA damage \{Costello 2013\} (3995), non-uniquely called sites (4719), germline SNVs

921 previously called by GATK HaplotypeCaller (591), probands with >20 mosaic variants (1490 in 10

922 probands), mosaic log posterior likelihood ratio $<10(940)$, variants with $>2$ parental alternative allele

923 reads (244), variants with gnomAD population frequency > 1e-4 or located in MUC or HLA genes (40).

Filtering of cardiovascular tissue Candidate Variants

We used the MosaicHunter pipeline in trio mode to identify candidate variants in WES data from

70 cardiovascular tissue samples (belonging to 66 unique probands). From the list of variants initially

928 reported by the pipeline using default settings, we applied the same filtration steps listed for

929 MosaicHunter candidate variants in blood samples with the exception of the removal of $\mathrm{G}$ to $\mathrm{T}$ mutations

930 with fewer than 10 alternative allele reads and the mosaic log posterior likelihood ratio < 10 . Finally, we

931 removed variants that were identified in either parent or had a total read depth $<10$ in either parent.

Clinical interpretation of mosaic variants - limitations

934 We note that conventional clinical interpretation of mosaic mutations is challenging for several

935 reasons: (i) it is unclear in which tissues each mosaic mutation is expressed (ii) several study participants

936 were very young at time of clinical assessment and many classical disease features may not yet have

937 developed or been noted, and (iii) the absence of additional clinical features does not necessarily rule out

938 a mosaic mutation as being for the cause of the CHD. For the purposes of this study, we selected these

939 mosaic mutations on the basis of predicted pathogenicity and detection in genes involved in biological

940 processes relevant to CHD or developmental disorders

942 Fig S1. EM-mosaic Flowchart. We first processed our SAMtools de novo calls using our upstream filters

943 ( $n=2396$ sites passing all filters). We then applied the same upstream filters to the published dnSNVs

944 from Jin et al. ( $n=2650$ sites passing all filters) before finally taking the union of these two call sets

945 ( $n=3192)$. High-confidence mosaics $(n=309)$ were defined as mosaics passing IGV inspection and having

946 posterior odds $>10$. Grey text indicates which filters removed candidate mosaic variants called by

947 MosaicHunter but not by EM-mosaic. 
949 Fig S2. Blood variants with posterior odds between 1 and 10.(A) Distribution of the 86 variants with

950 posterior odds between 1 and 10.(B) Histogram of counts by bin. To estimate the number of potential

951 mosaics mosaics missed by our threshold, counts of each bin were scaled by the estimated true positive

952 rate (TPR; posterior odds / 1+posterior odds). By our estimate, 54/86 variants were likely mosaic and

$953 \quad 32 / 86$ were likely germline.

Fig S3. MosaicHunter workflow. Quality Control filters excluded any sites that were (1) present in ExAC

(2) $G>T$ with $N_{\text {alt }}<10$ (3) parent $N_{\text {alt }}>2$. Outliers were defined as probands carrying more than 20 mosaics,

957 or non-unique sites. We also removed sites called as germline by GATK Haplotype Caller. High-

confidence mosaics $(n=116)$ were defined as having Likelihood Ratio $>80$ and affecting coding regions excluding MUC/HLA genes. Grey text indicates which filters removed variants called by EM-mosaic but not by MosaicHunter.

Fig S4. Comparison of variant allele fraction (VAF) and read depth of EM-mosaic and

963 MosaicHunter. Candidate mosiac variants detected by the two pipelines had more overlapping variants 964 at low read depth and VAF values.

Fig S5. Targeted sequencing to validate candidate blood mosaic variants. (A) EM-mosaic and (B)

967 MosaicHunter variants were assayed using PCR followed by MiSeq for high-depth assessment of

968 mosaicism. Variants with $\mathrm{x}$ symbols were shared by both pipelines. Mosaic variants that validated are

969 black, while variants with VAF $>0.45$ and therefore germline are red.

970 Validation VAF values demonstrated significant correlation with the original WES-derived VAF for EM-

971 mosaic (Pearson's correlation $\left.P=2.2 \times 10^{-16}\right)$ and MosaicHunter $\left(P=8.2 \times 10^{-11}\right)$.

973 Fig S6. Overdispersion. Overdispersion is commonly seen in WES data \{Heinrich 2012; Ramu 2013\}

974 and is defined as observing variance (in terms of the VAF of variants with a given DP value) higher than 
975 expected across DP values, under a given statistical model. The blue line denotes the expectation under

976 a Binomial model and the red line denotes the expectation under a Beta-Binomial model.

978 Fig S7. FDR-based minimum $\mathbf{N}_{\text {alt }}$ threshold. A FDR-based approach was used to determine a threshold

979 for the minimum number of reads supporting the alternate allele for each site to avoid false positives

980 caused purely by sequencing errors. Assuming that sequencing errors are independent and that errors

981 occur with probability 0.005 , with the probability of an allele-specific error being $0.005 / 3=0.00167$, and

982 given the total number of reads $(M)$ supporting a variant site, we iterated over a range of possible $N_{\text {alt }}$

983 values between 1 and $0.5^{\star} \mathrm{N}$ and estimated the expected number of false-positives due to sequencing

984 error, exome-wide $\left(\left(1-f_{\text {poisson }}\left(\mathrm{x}=n, \lambda=N^{\star} 0.005 / 3\right)\right)^{\star} 3 \times 10^{7}\right.$; where $f_{\text {poisson }}$ is the probability of $\mathrm{x}$ events in a

985 Poisson process with mean $\lambda$ ). Assuming one coding de novo SNV per exome \{Acuna-Hidalgo 2016\} and

986 that roughly $10 \%$ of de novo SNVs arise post-zygotically \{Lim 2017; Krupp 2017; Freed 2016\}, we used a

987 conservative assumption of 0.1 mosaic mutation per exome. To constrain theoretical FDR to $10 \%$ we

988 allowed a maximum of 0.01 false positives per exome and used the corresponding $N_{\text {alt }}$ value to define an

989 FDR-based minimum $N_{\text {alt }}$ threshold for each variant. We then excluded variants with alternate allele read

990 counts below this threshold.

992 Fig S8. Estimated mosaic detection power using less stringent mosaic definitions. (A) Estimated

993 true frequency of detectable coding mosaics $(0.4>$ VAF $>0.1)$ adjusted by detection power $(n=341$;

994 0.135/exome) (B) Calibrated mosaic detection power and estimated true mosaic frequency of detectable

995 coding mosaics, using posterior odds cutoff of $5(n=361 ; 0.143 /$ exome). (C) Calibrated mosaic detection

996 power and estimated true mosaic frequency of detectable coding mosaics, using posterior odds cutoff of

$9972(0.4>\mathrm{VAF}>0.1 ; \mathrm{n}=424 ; 0.168 /$ exome $)$.

998

999 Fig. S9. Mosaic variants shared in blood and cardiovascular tissues have higher variant allele

1000 fraction. Validation VAF from (A) cardiovascular tissue and (B) blood had higher VAF for shared variants 1001 compared to tissue-specific variants ( $p=0.101$ and 0.015 , respectively). 
Fig. S10. Damaging CHD-related mosaics have higher VAF under less stringent definitions of mosaicism. (A) Using posterior odds cutoff of 5 (corresponding to 315 mosaics). Among 78 mosaics in CHD-related genes (left), there were 14 variants predicted as damaging, 63 variants predicted as likelybenign, and 1 variant of unknown functional consequence. Among 237 mosaics in non-CHD-related genes (right), there were 41 variants predicted as damaging, 184 variants predicted as likely-benign, and

10082 variants of unknown functional consequence. (B) Using posterior odds cutoff of 2 (corresponding to 352 1009 mosaics). Among 89 mosaics in CHD-related genes (left), there were 17 variants predicted as damaging, 101071 variants predicted as likely-benign, and 1 variant of unknown functional consequence. Among 263 1011 mosaics in non-CHD-related genes (right), there were 54 variants predicted as damaging, 206 variants 1012 predicted as likely-benign, and 3 variants of unknown functional consequence.

1014 Fig. S11. Mosaic rate by age. (A) Age distribution for all 2530 probands in cohort. (B) Mosaic Rate 1015 across Age ranges. Rate = \# mosaics/\# probands in age bin. Note: 9/2530 probands missing Age 1016 information. 1/367 mosaic belong to a proband with missing Age.

Fig. S12. Mosaic rate by parental age at birth. Mosaic rate by age of father (blue) and mother (red) at birth. Rate $=$ \# mosaics/\# probands in each parental age bin. Note: 9/2530 probands missing age

1020 information. 1/367 mosaic belong to a proband with missing age.

Fig. S13. Confirmation rate across VAF bins. The number of candidates for which we performed MiSeq resequencing among $(\mathbf{A})$ the union set $(n=143$ tested) $(B)$ all EM-mosaic calls $(n=97)$ and $(\mathbf{C})$ all

1024 MosaicHunter $(n=68)$ calls vs. the number confirmed as mosaic for VAF ranges $[0,0.1),[0.1,0.2)$, and $1025[0.2,0.3)$.

1027 Fig. S14. Posterior odds comparison for tested vs. untested mosaics. Among 309 candidates with 1028 EM-mosaic posterior odds scores available, we compared the distribution of tested $(n=97)$ vs. untested $1029(\mathrm{n}=212)$ mosaics. The $\log _{10}$-scaled posterior odds distribution for the tested group is shown in blue 1030 (mean=5.382). The $\log _{10}$-scaled mean posterior odds for the untested group is shown in red 
1031 (mean=7.050). The selected candidates had lower posterior odds than those not selected for

1032 confirmation (Mann Whitney $U$ test $P=0.002$ ).

1033

1034 Table S1. Proband Phenotypes. Cardiac and neurodevelopmental phenotypes for CHD probands. NDD

1035 diagnosis is unknown for patients $<1$ year of age.

1036

1037 Table S2. Cohort Summary. Number of de novo and mosaic variants for probands with isolated CHD,

1038 extracardiac anomalies (ECA), neurodevelopmental delay (NDD) or unknown phenotypes.

1039

1040

Table S3. EM-mosaic Mosaic Candidates. Candidate mosaic variants identified by EM-mosaic and

1041 MiSeq validation results.

1042

1043

Table S4. MosaicHunter Mosaic Candidates. Candidate mosaic variants identified by MosaicHunter

1044 and MiSeq validation results.

1045

1046 Table S5. Blood mosaic Candidate Validation by MiSeq. 143 candidate mosaic sites were assessed 1047 using targeted PCR and deep sequencing. 85/97 (88\%) of selected EM-mosaic sites and 44/68 (67\%) of 1048 selected MosaicHunter sites were confirmed.

Table S6. Cardiovascular Tissues with Whole Exome Sequencing. 70 tissues from 66 CHD probands 1051 were assessed for mosaic variants.

Table S7. CHD tissue mosaic Candidate Validation by MiSeq. 24 candidate mosaic sites were assessed using targeted PCR and deep sequencing. 85/92 (92\%) of selected EM-mosaic sites and 44/64

1055 (69\%) of selected MosaicHunter sites were confirmed.

1057 Table S8. CHD related genes. We considered the union of genes highly expressed in the developing 1058 heart (HHE) and known candidate CHD genes $\{$ Jin 2017\} as CHD-related genes $(n=4558)$. 
bioRxiv preprint doi: https://doi.org/10.1101/733105; this version posted August 13,2019 . The copyright holder for this preprint (which was not certified by peer review) is the author/funder, who has granted bioRxiv a license to display the preprint in perpetuity. It is made available under aCC-BY-NC-ND 4.0 International license.

1060 Table S9. All Protein-Altering Mosaics. Detailed information for 398 mosaic variants predicted to affect 1061 protein sequence.

1062

1063 Table S10. Damaging Mosaics in CHD-Relevant Genes. Detailed information for 25 mosaic variants 1064 likely to contribute to CHD.

1065

1066 


\begin{tabular}{|c|c|c|c|c|c|c|c|c|c|c|c|}
\hline \multirow{2}{*}{ ID } & \multirow{2}{*}{ Gene } & \multirow{2}{*}{$\begin{array}{c}\text { Variant } \\
\text { Class }\end{array}$} & \multirow{2}{*}{ Pipeline } & \multicolumn{4}{|c|}{ CHD Tissue } & \multicolumn{3}{|c|}{ Blood WES VAF } & \\
\hline & & & & Location & WES AD & WES VAF & MiSeq VAF & WES AD & WES VAF & MiSeq V & $A F_{0}^{0} \frac{0}{9}$ \\
\hline $1-00543$ & CTCFL & Bmis & EM-mosaic & $\mathrm{AO}$ & 138,36 & 0.21 & 0.32 & 29,8 & 0.22 & 0.19 & \\
\hline $1-00984$ & ZNF16 & syn & EM-mosaic & LV & 262,1 & 0.00 & 0.01 & 100,7 & 0.07 & 0.07 & \\
\hline $1-01282$ & GABRA6 & Dmis & MosaicHunter & $\mathrm{RV}$ & 104,1 & 0.01 & 0.01 & 55,12 & 0.18 & 0.18 & 案 \\
\hline $1-01684$ & CCNC & Bmis & Both & AoValve, RV & 36,7 & 0.16 & $0.17,0.19$ & 224,40 & 0.15 & 0.14 & \\
\hline $1-02672$ & TOR1A & syn & Both & AtrSpt & 159,10 & 0.06 & 0.10 & 29,6 & 0.17 & 0.19 & \\
\hline $1-03512$ & RFX3 & LoF & MosaicHunter & RV & 156,15 & 0.09 & 0.08 & 39,0 & 0.00 & 0.03 & \\
\hline $1-04652$ & PCDH10 & syn & Both & AtrSpt & 154,19 & 0.11 & 0.14 & 15,1 & 0.06 & 0.10 & \\
\hline $1-07004$ & ANK2 & Bmis & MosaicHunter & SubAoMembr & 226,13 & 0.05 & 0.04 & 30,0 & 0.00 & 0.00 & 咅总 \\
\hline $1-07004$ & MYH14 & Bmis & Both & SubAoMembr & 124,22 & 0.15 & 0.27 & 33,0 & 0.00 & 0.00 & इ屯े \\
\hline $1-07004$ & NRG3 & Bmis & EM-mosaic & SubAoMembr & 152,30 & 0.16 & 0.24 & 43,0 & 0.00 & 0.00 & 总 \\
\hline $1-07004$ & NUDT21 & Bmis & Both & SubAoMembr & 137,22 & 0.14 & 0.14 & 74,0 & 0.00 & 0.02 & 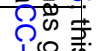 \\
\hline $1-07004$ & TET3 & Dmis & MosaicHunter & SubAoMembr & 131,1 & 0.01 & 0.03 & 81,16 & 0.16 & 0.27 & 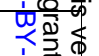 \\
\hline $1-07299$ & $R R S 1$ & syn & Both & RV, UNK & 160,25 & 0.14 & 0.25 & 22,2 & 0.08 & 0.14 & 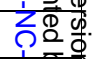 \\
\hline $1-09869$ & PIK3C2G & LoF & MosaicHunter & LV & 126,9 & 0.07 & 0.10 & 31,0 & 0.00 & 0.00 & 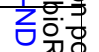 \\
\hline $1-11800$ & TMEM45A & Bmis & MosaicHunter & RV & 213,0 & 0.00 & 0.00 & 32,7 & 0.18 & 0.06 & $\begin{array}{l}x \\
\frac{x}{<} \cdot \frac{p}{1} \\
\end{array}$ \\
\hline $\begin{array}{l}1067 \\
1068 \\
1069 \\
1070 \\
1071 \\
1072 \\
1073\end{array}$ & $\begin{array}{l}\text { Table 2. Mo } \\
\text { Characteristic } \\
\text { analysis of bl } \\
\text { of blood were } \\
\text { absent from C } \\
\text { septum; Bmis }\end{array}$ & $\begin{array}{l}\text { aics detec } \\
\text { of mosaic } v \\
\text { d WES, } 8 \text { u } \\
\text { resent in bo } \\
\text { HD tissue W } \\
\text { benign miss }\end{array}$ & $\begin{array}{l}\text { in individuals } \\
\text { iants predicted for } \\
\text { re detected from ca } \\
\text { DNA sources with } \\
\text { S. Minimum } 1023 \mathrm{M} \\
\text { nse; Dmis, deleterio }\end{array}$ & $\begin{array}{l}\text { th matched card } \\
\text { ividuals with blood } \\
\text { ovascular tissue W } \\
\text { Seq VAF } \geq 0.01 \text {. Two } \\
\text { eq reads used to de } \\
\text { missense; LOF, Lo }\end{array}$ & $\begin{array}{l}\text { ascular tis } \\
\text { cardiovascul } \\
\text { and } 2 \text { were d } \\
\text { ditional varia } \\
\text { ine VAF. Ab } \\
\text { f function var }\end{array}$ & $\begin{array}{l}\text { and blood } \\
\text { ssue WES data } \\
\text { sted by both ap } \\
\text { previously iden } \\
\text { iations: AD, all } \\
\text {; LV, left ventri }\end{array}$ & $\begin{array}{l}\text { aailable. Among } 1 \\
\text { aches. Six of } 7 \text { ( } \\
\text { ed as de novo ge } \\
\text { c depth (referenc } \\
\text {; RV, right ventric }\end{array}$ & $\begin{array}{l}\text { mosaics, } 5 \mathrm{w} \\
\% \text { \%) mosaics } 0 \\
\text { line variants i } \\
\text { alternate); AC } \\
\text {; VAF, variant }\end{array}$ & $\begin{array}{l}\text { detected via } \\
\text { ected from anal } \\
\text { lood WES wer } \\
\text { aorta; AtrSpt, a } \\
\text { lele fraction }\end{array}$ & & 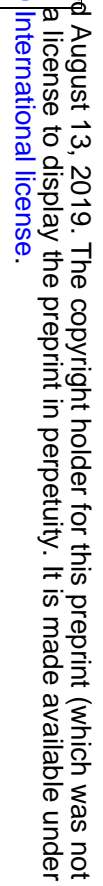 \\
\hline
\end{tabular}




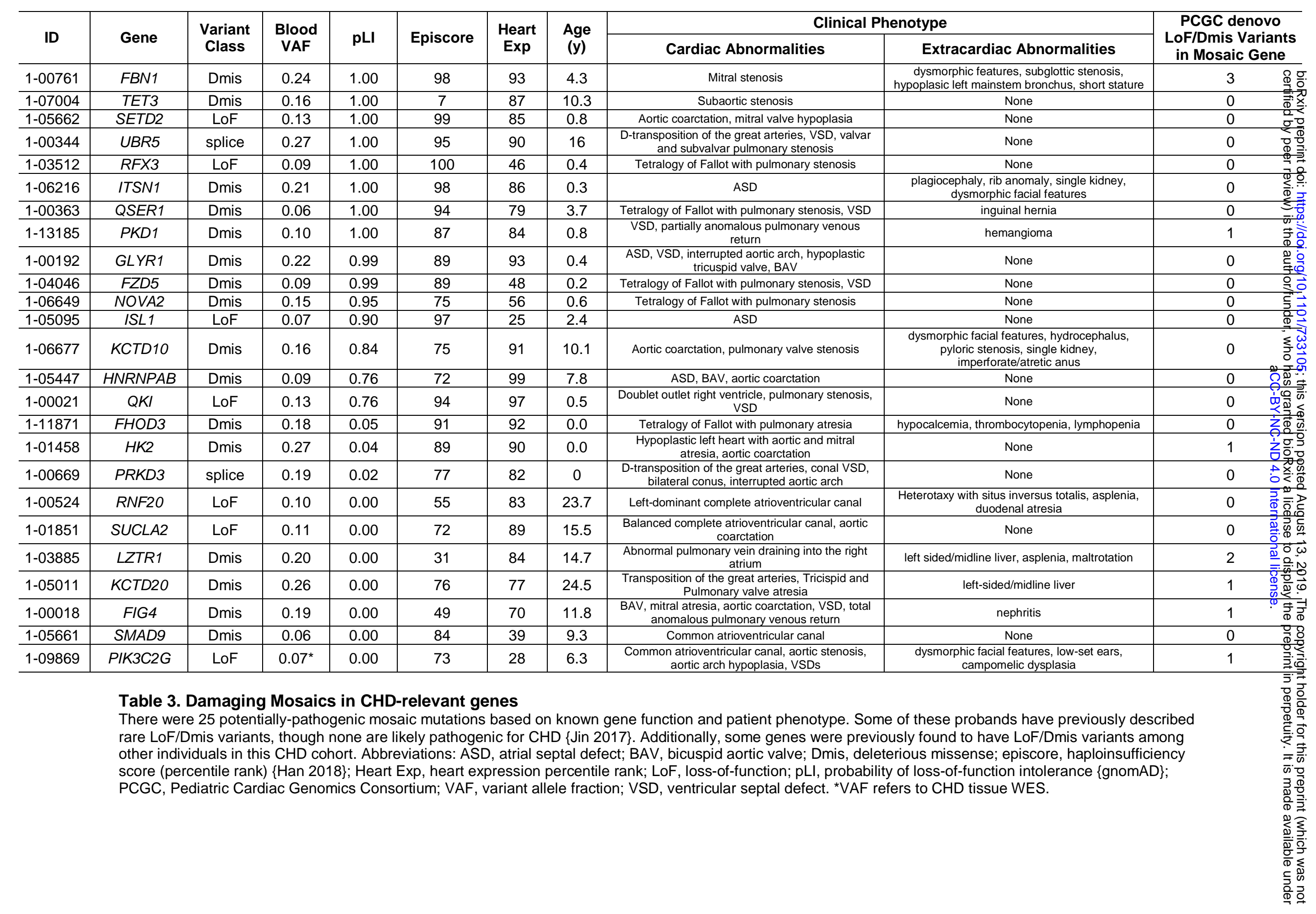




\section{Declarations}

Ethics approval and consent to participate

CHD subjects were recruited to the Congenital Heart Disease Network Study of the Pediatric Cardiac Genomics Consortium (CHD GENES: ClinicalTrials.gov identifier NCT01196182). The institutional review boards of Boston's Children's Hospital, Brigham and Women's Hospital, Great Ormond Street Hospital, Children's Hospital of Los Angeles, Children's Hospital of Philadelphia, Columbia University Medical Center, Icahn School of Medicine at Mount Sinai, Rochester School of Medicine and Dentistry, Steven and Alexandra Cohen Children's Medical Center of New York, and Yale School of Medicine approved the protocols. All subjects or their parents provided informed consent.

\section{Consent for publication}

See above.

\section{Availability of data and material}

EM-mosaic and code for analyzing data are available from https://github.com/ShenLab/mosaicism. The MosaicHunter software is available from http://mosaichunter.cbi.pku.edu.cn/. SAMtools is available from http://www.htslib.org/. ANNOVAR is available from http://annovar.openbioinformatics.org/en/latest/. Integrative Genomics Viewer (IGV) software is available from https://software.broadinstitute.org/software/igv/. Whole-exome sequencing data have been deposited in the database of Genotypes and Phenotypes ( $\mathrm{dbGaP}$ ) under accession numbers phs000571.v1.p1, phs000571.v2.p1 and phs000571.v3.p2. Inhouse pipelines are available from the corresponding authors on reasonable request. 


\section{Competing interests}

The authors declare that they have no competing interests.

\section{Funding}

This work was supported by the National Heart, Lung, and Blood Institute (NHLBI) grants for the Pediatric Cardiac Genomics Consortium [U01-HL098188, U01HL131003, UM1-HL098147, U01-HL098153, U01-HL098163, UM1-HL098123, UM1HL098162, UM1-HL128761, UM1-HL128711].

\section{Authors' contributions}

YS, JGS, CES, and WKC conceived and oversaw the study. AH, SUM, JALW, HQ, KBM, JGS, CES, YS, WKC analyzed the data. AH developed the EM-mosaic pipeline and wrote the statistical analysis code. SUM, JALW carried out MosaicHunter analyses of blood and tissue samples. JMG, AT, SD performed MiSeq experimental confirmation. AH, SUM, EG, CES, WKC interpreted the impact of mosaics on participant clinical phenotypes. DB, RWK, JWN, GAP, DS, MT-F, MB, RPL, EG, BDG, CES, JGS, WKC were involved in cohort ascertainment, phenotypic characterization, and recruitment. DM collected cardiovascular tissue samples. AH, SUM, JALW, YS, JGS, CES, WKC wrote the manuscript. All authors read and approved the manuscript.

\section{Acknowledgements}

The authors would like to thank all the participants and their families. 
Fig 1

A

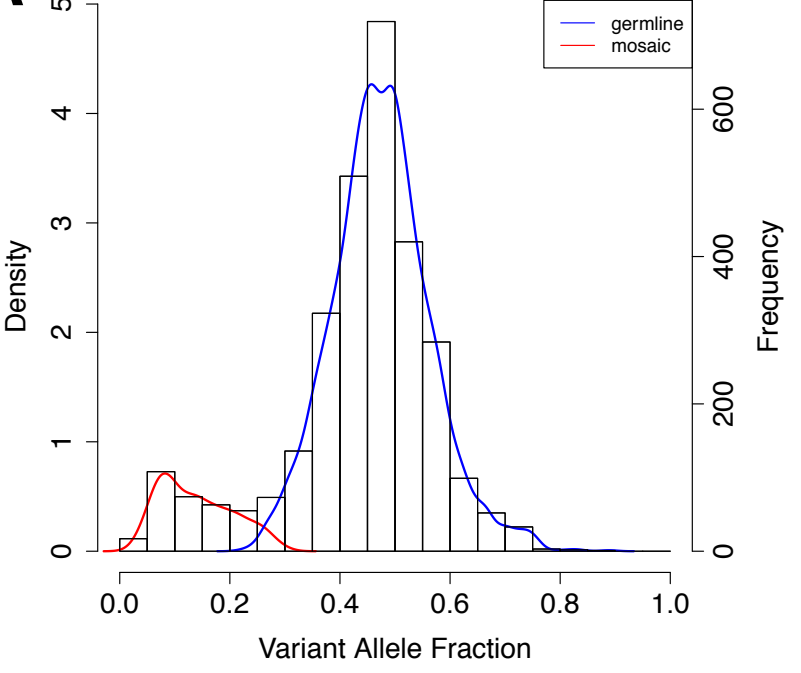

B

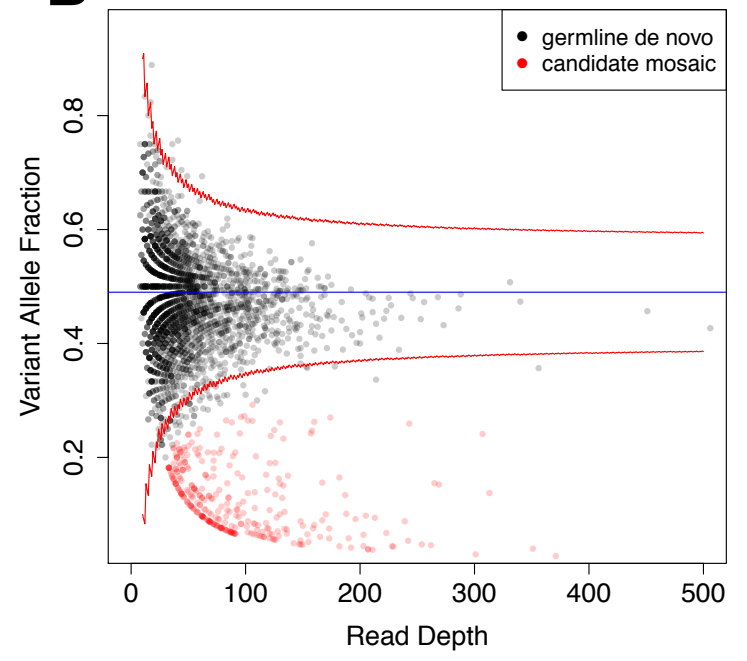

C

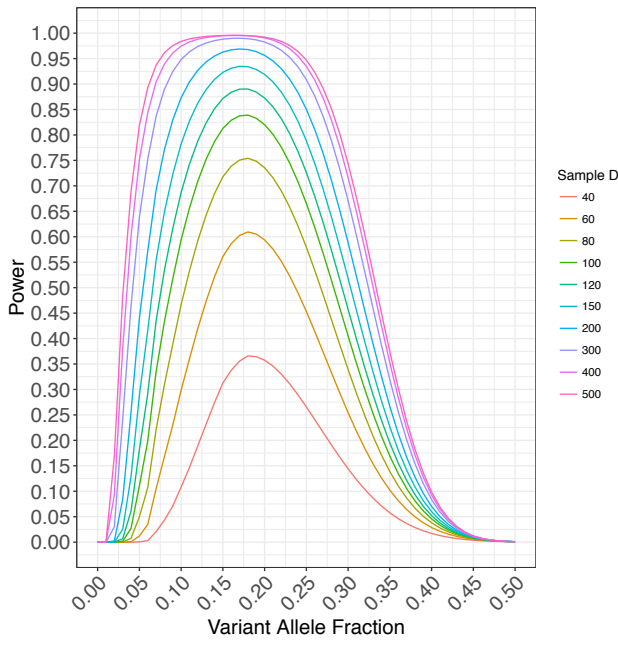


Fig 3

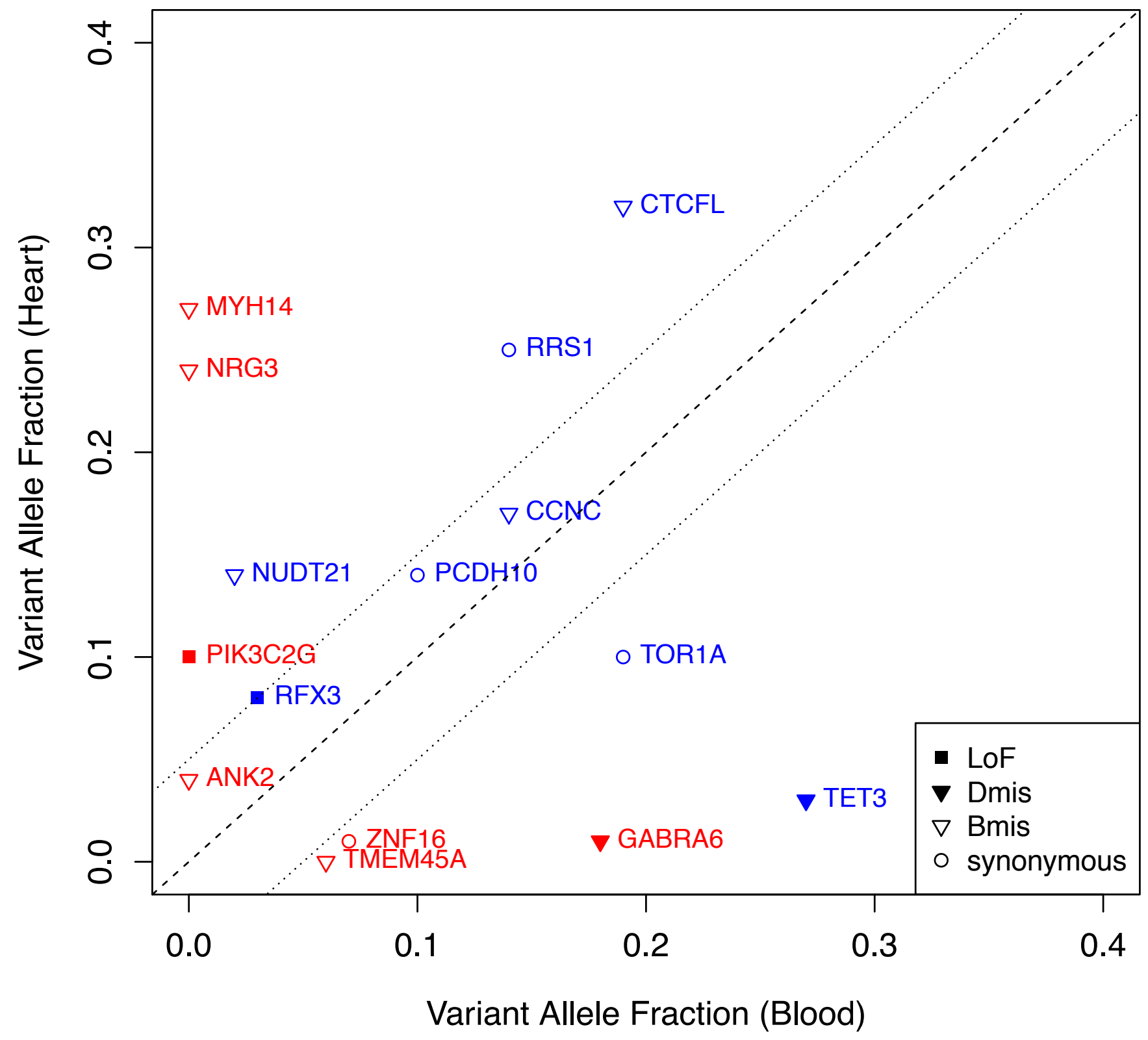


Fig 4

A

Likely Benign-

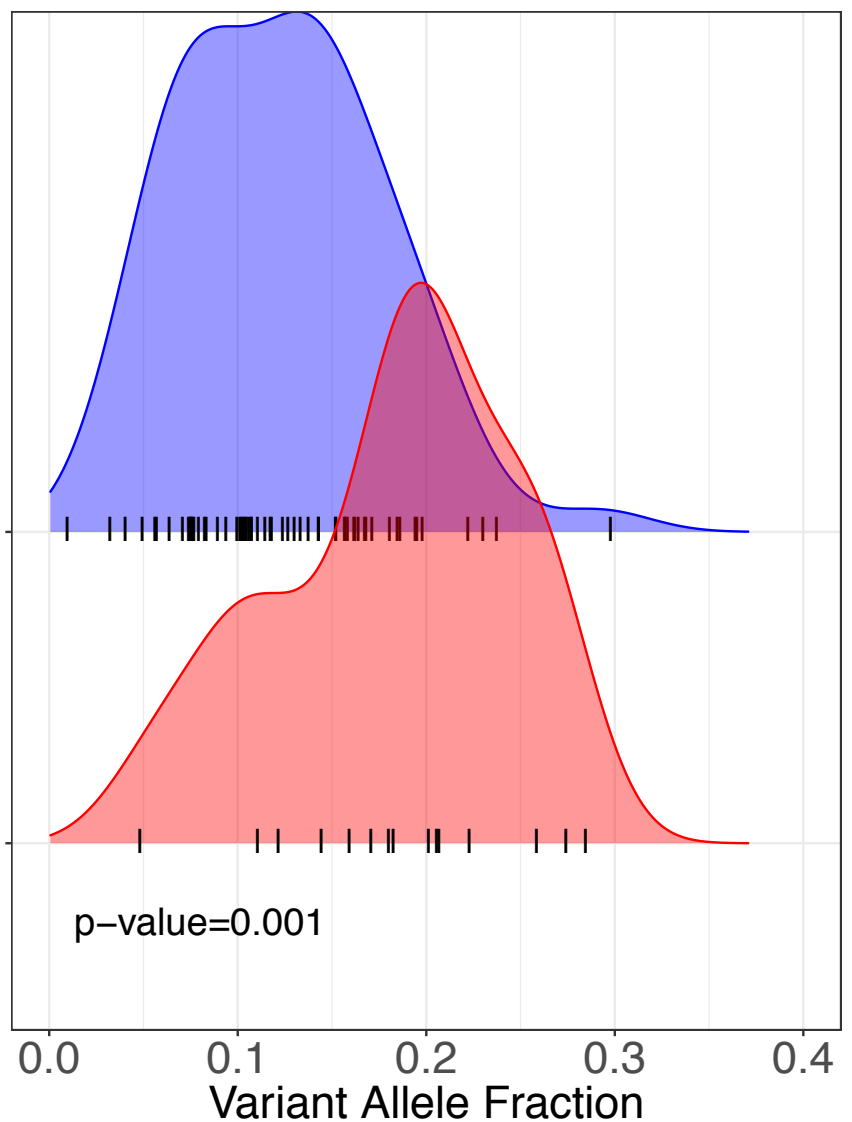

B

Likely Benign

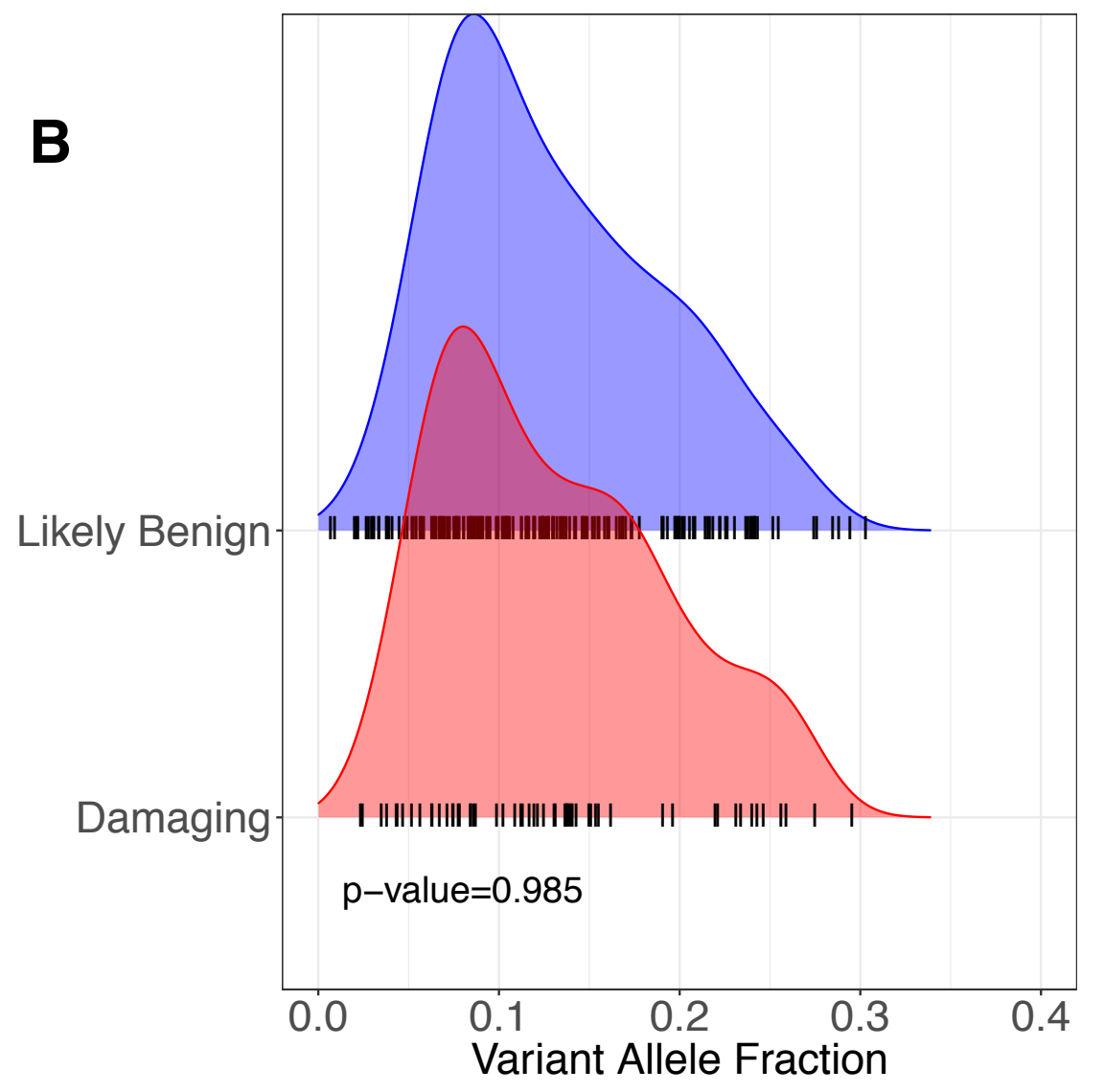

Damaging

Variant Allele Fraction 
Parse validated DNMs from Jin et al.
Call de novo SNVs using

SAMtools with settings

optimized for mosaic sites

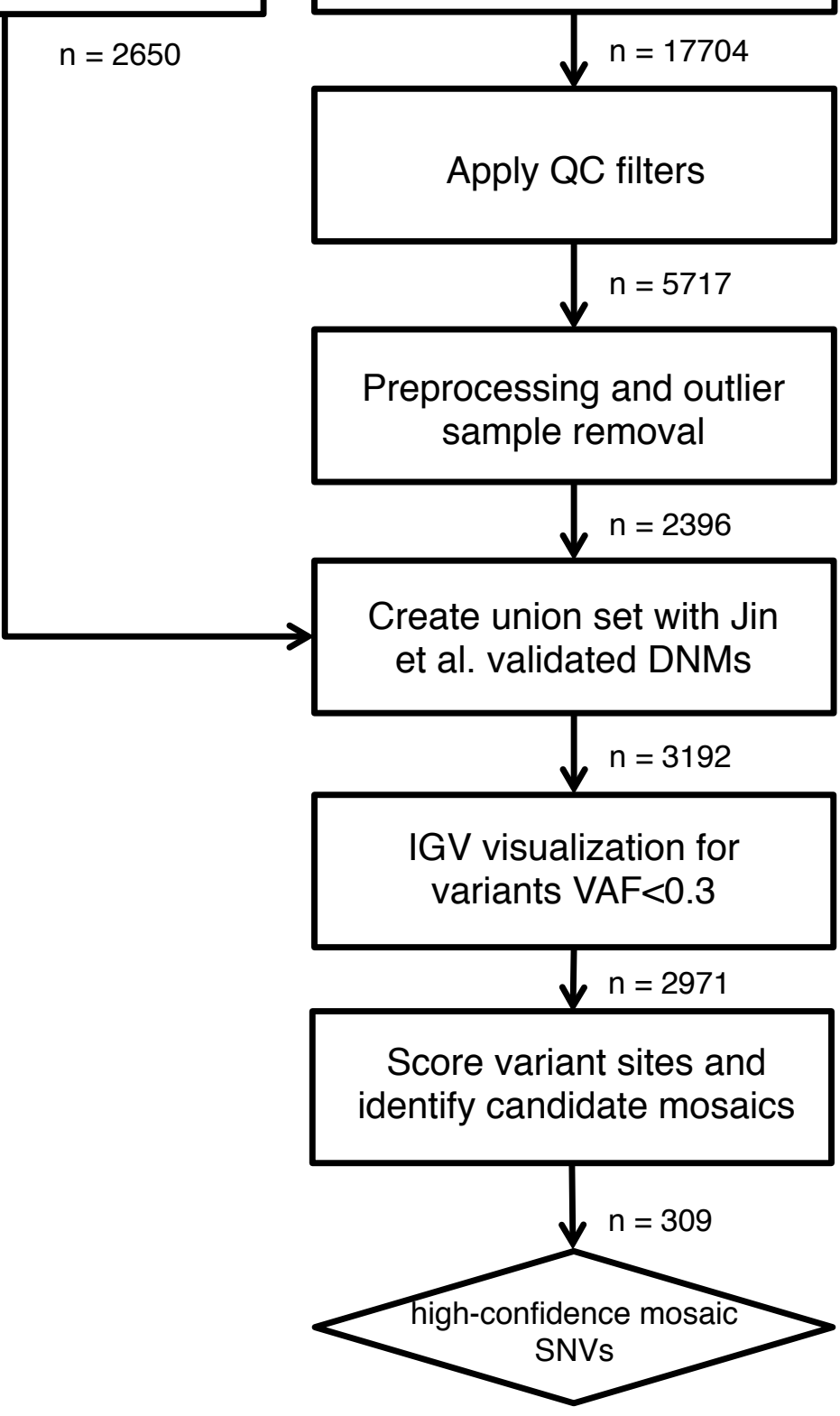

- $28 \mathrm{MH}$ variants not called

- $11 \mathrm{MH}$ variants removed due to parent alt reads $>0$

- $5 \mathrm{MH}$ variants removed for other quality filters 
Fig S2

A

\section{B}
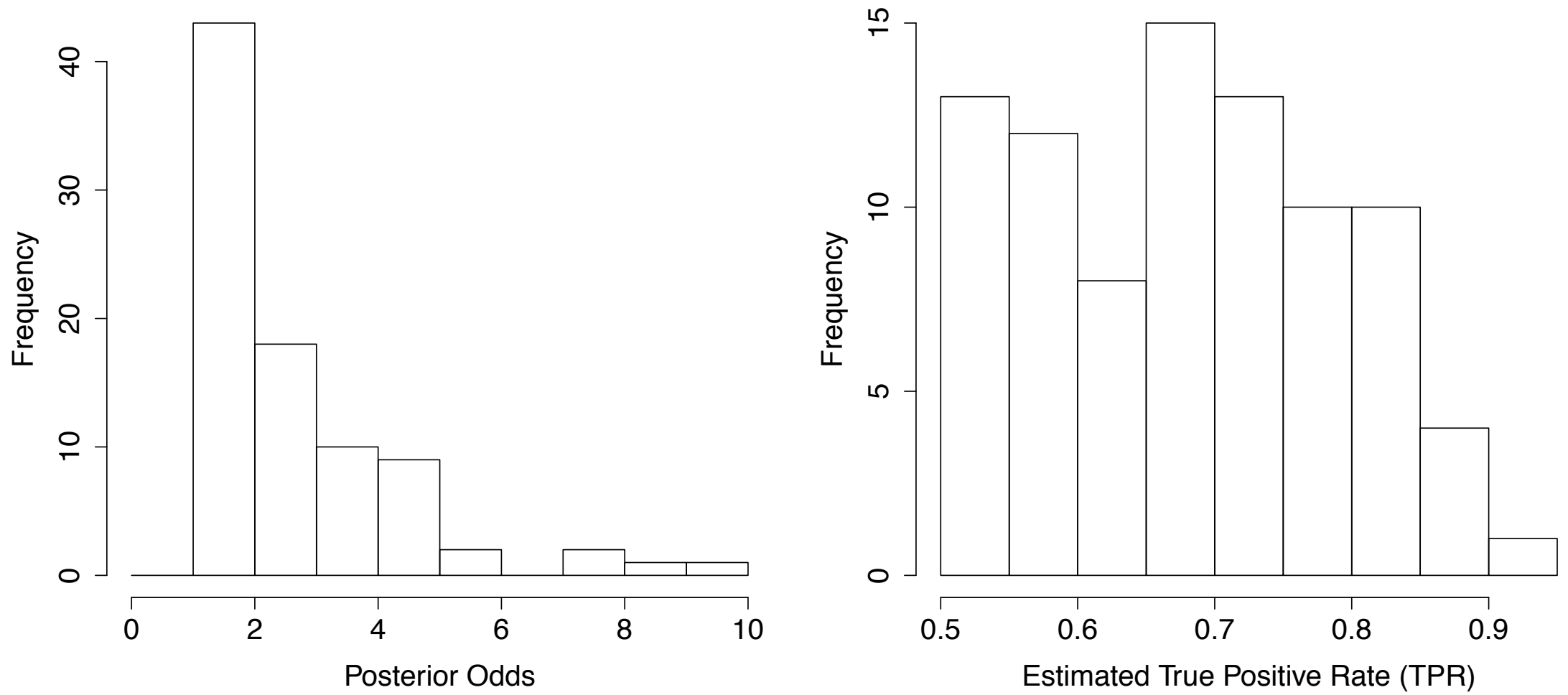
Fig S3

Call mosaic SNVs using

MosaicHunter

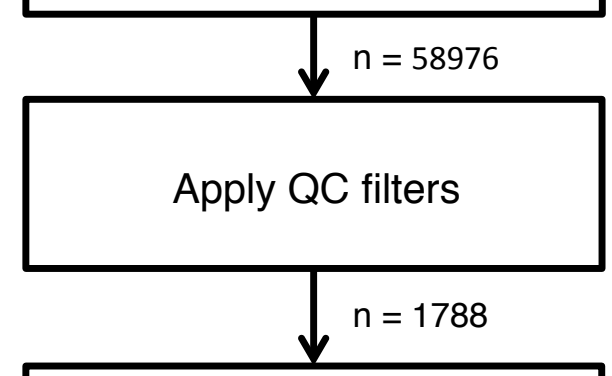

Outlier sample removal

$\downarrow n=1727$

Remove germline sites called by GATK

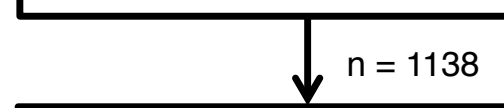

Apply Likelihood Ratio

(LR) cutoff

\rfloor$^{n}=350$

Select coding variants

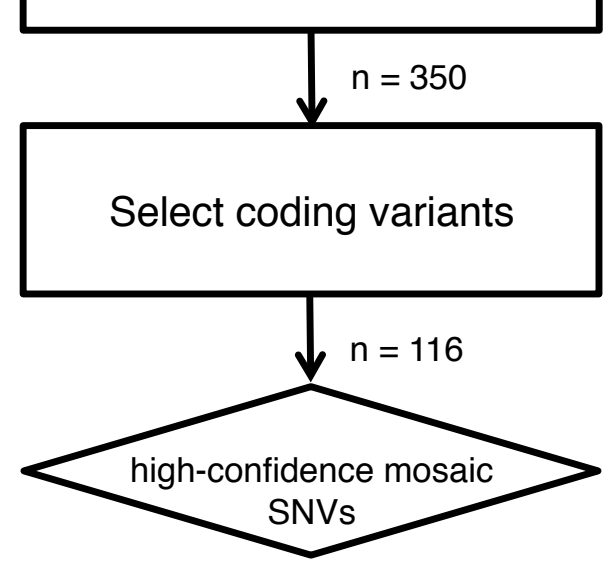

- 70 EZ-mosaic variants not called

- 13 EZ-mosaic variants removed in BAM filtering

- $141 \mathrm{EZ}-\mathrm{mosaic}$ variants removed in MH quality filters

- 4 EZ-mosaic variants removed by $\mathrm{G}>\mathrm{T}<6$

- 1 EZ-mosaic variants in >20 mosaics proband 
Fig S4

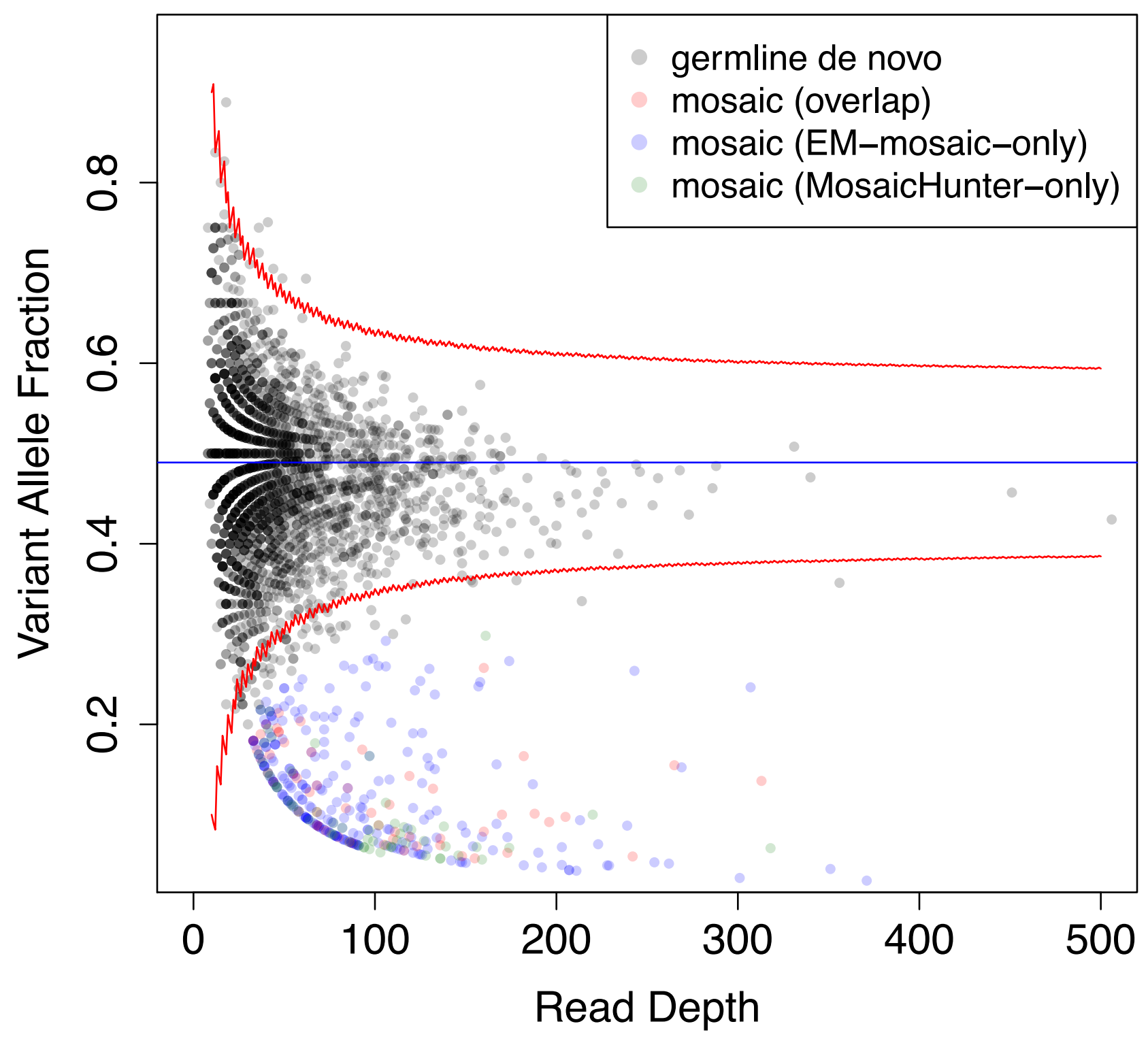


Fig S5

A

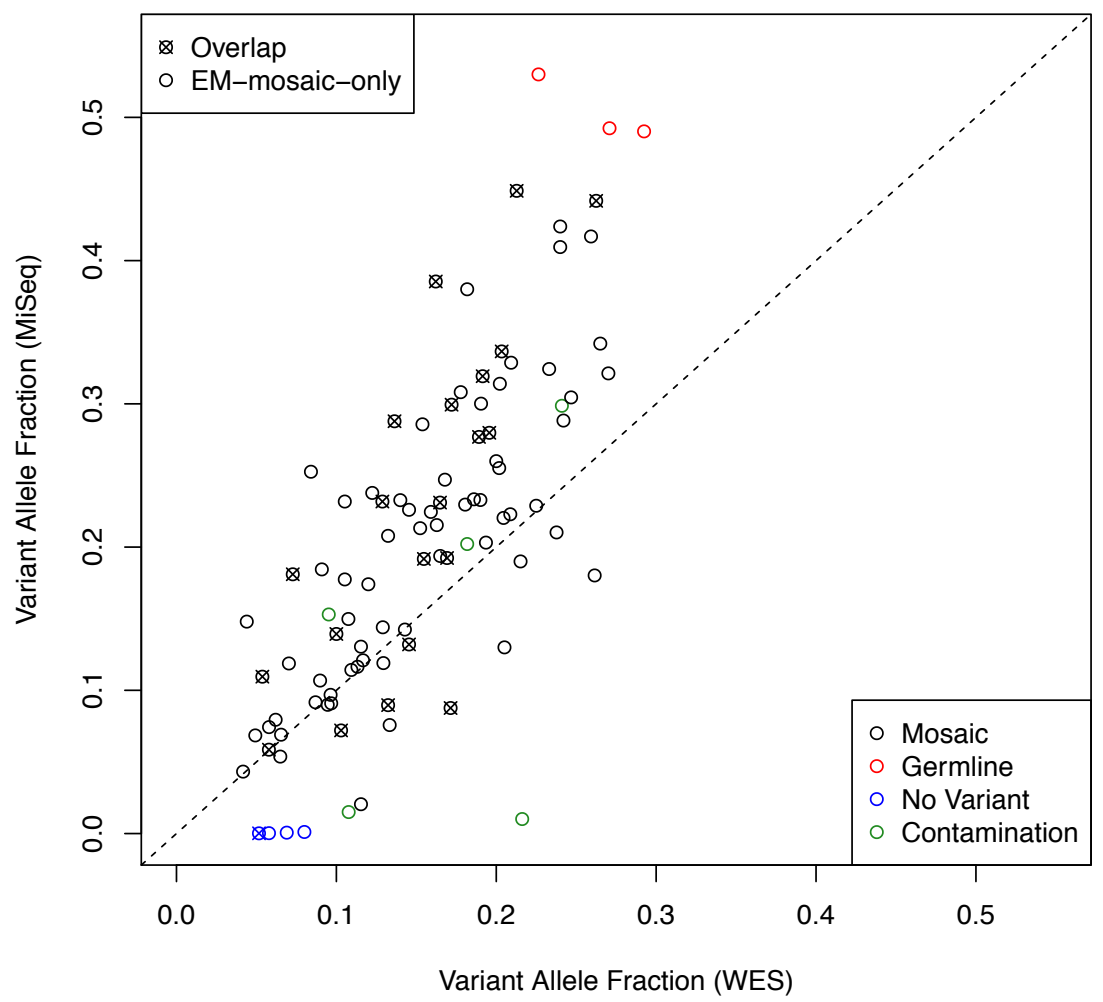

B

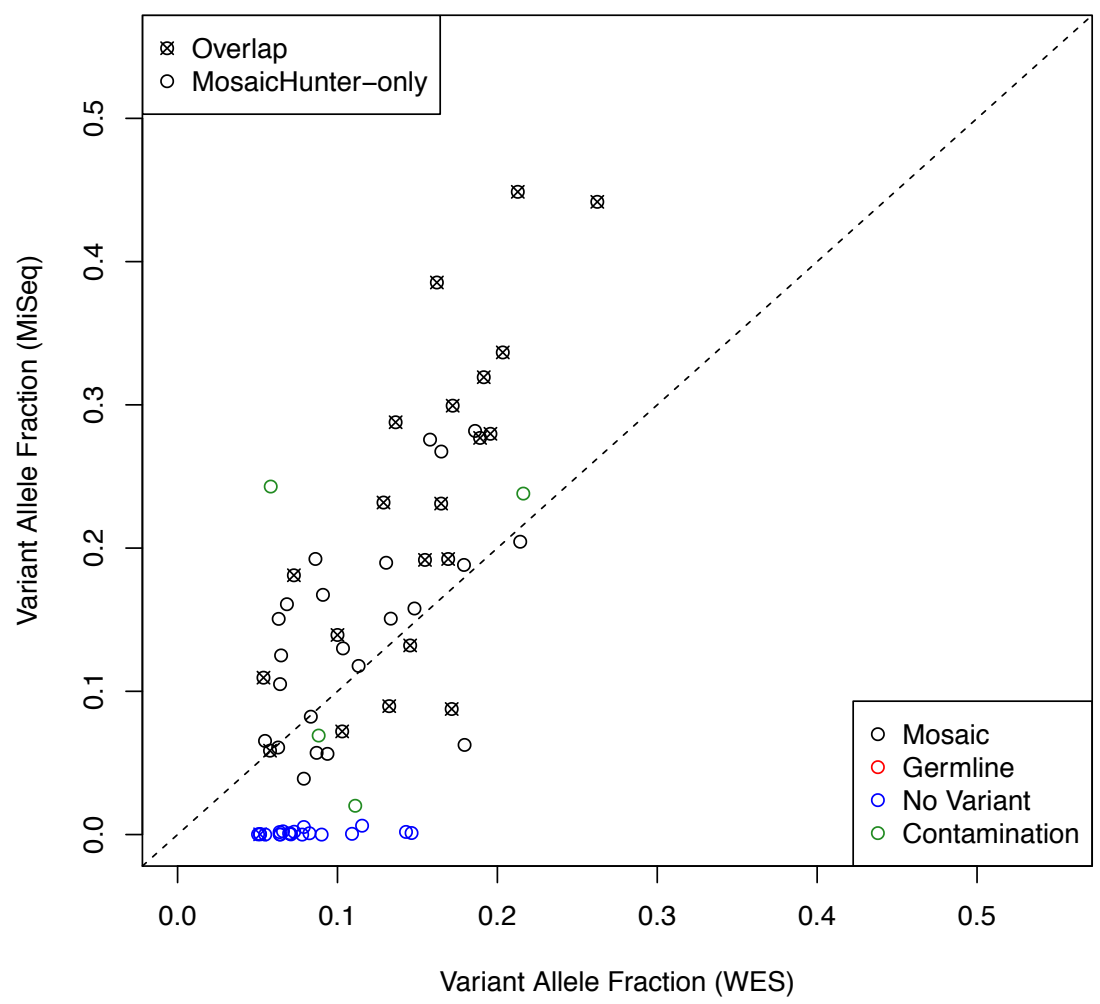


Fig S6

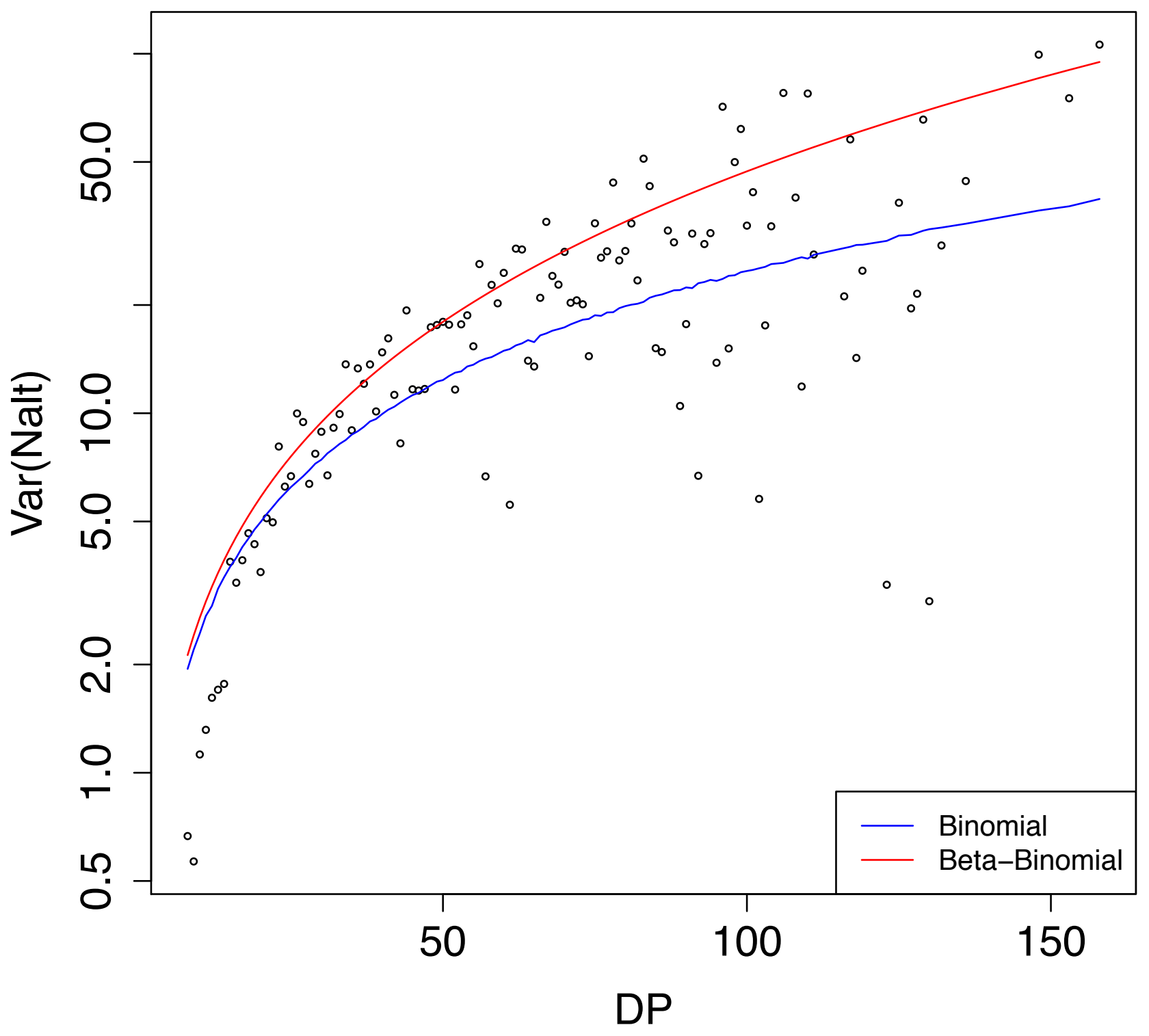


Fig S7

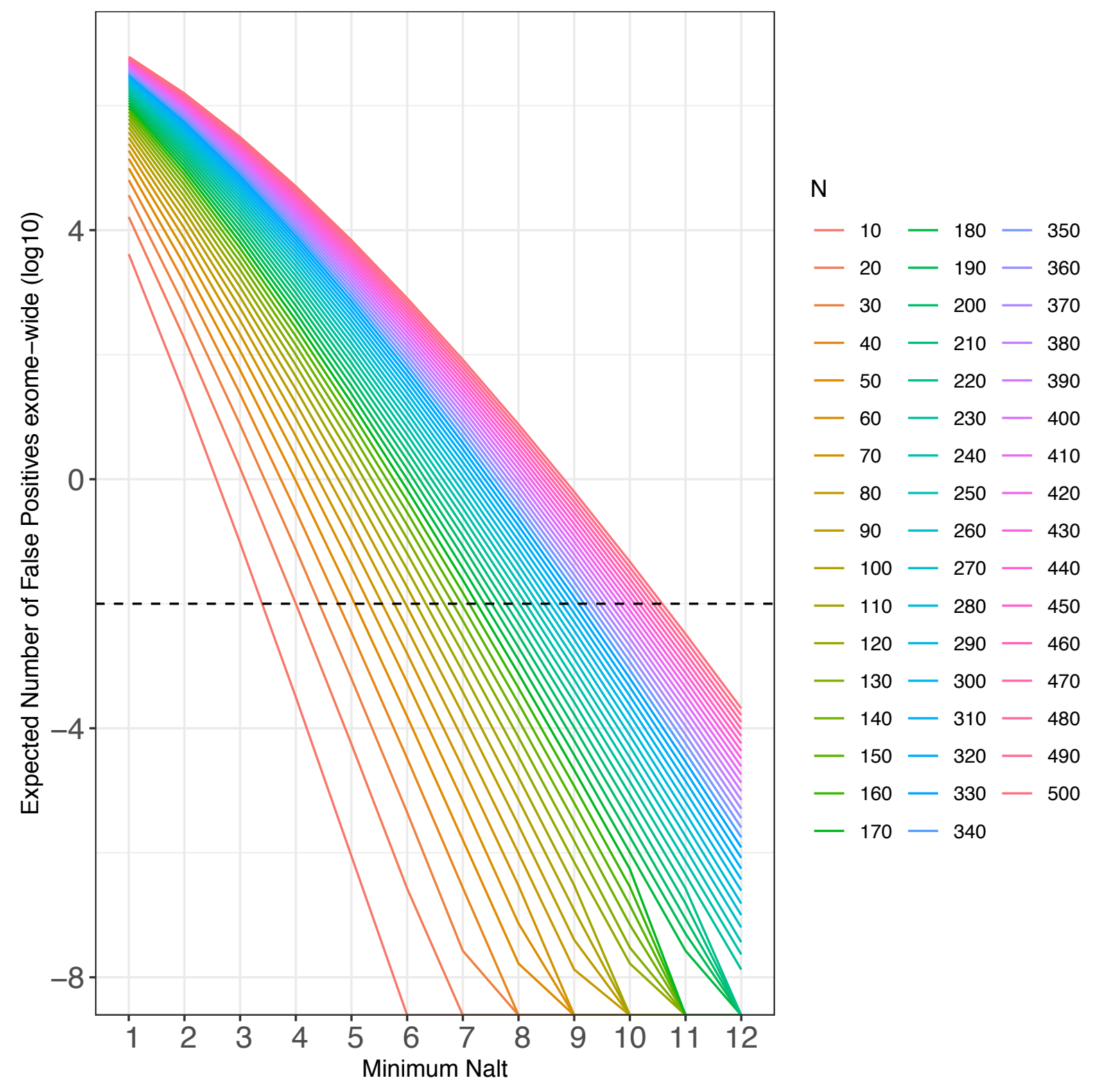


A

Fig S8
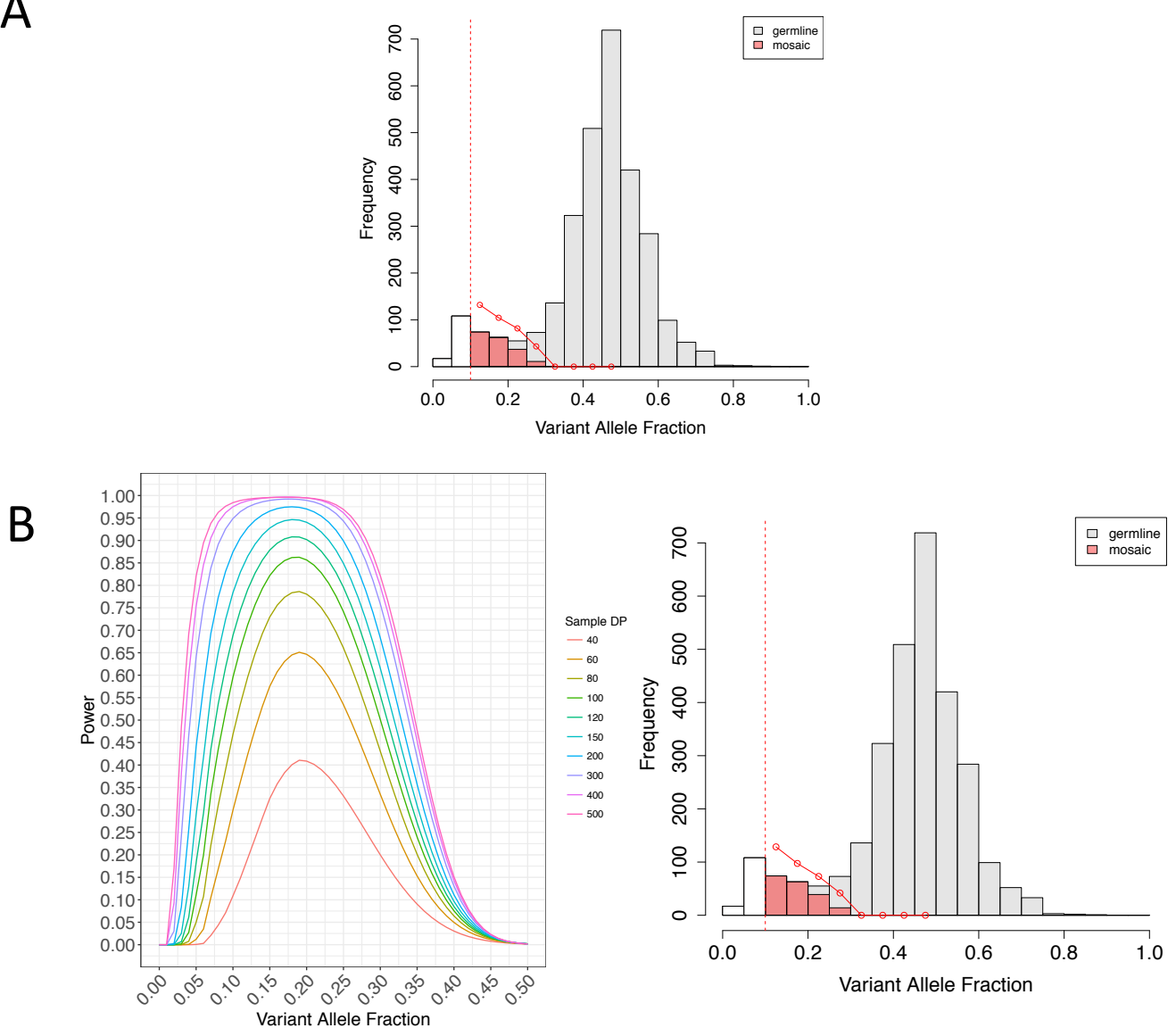

C
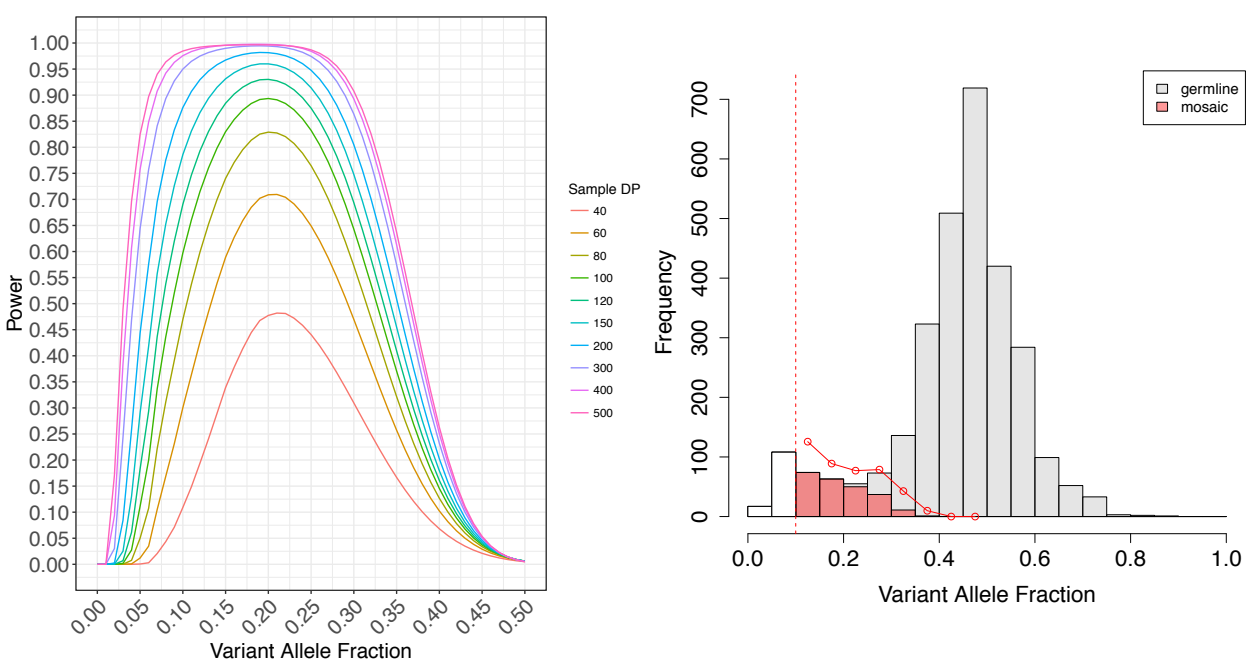


\section{Fig S9}
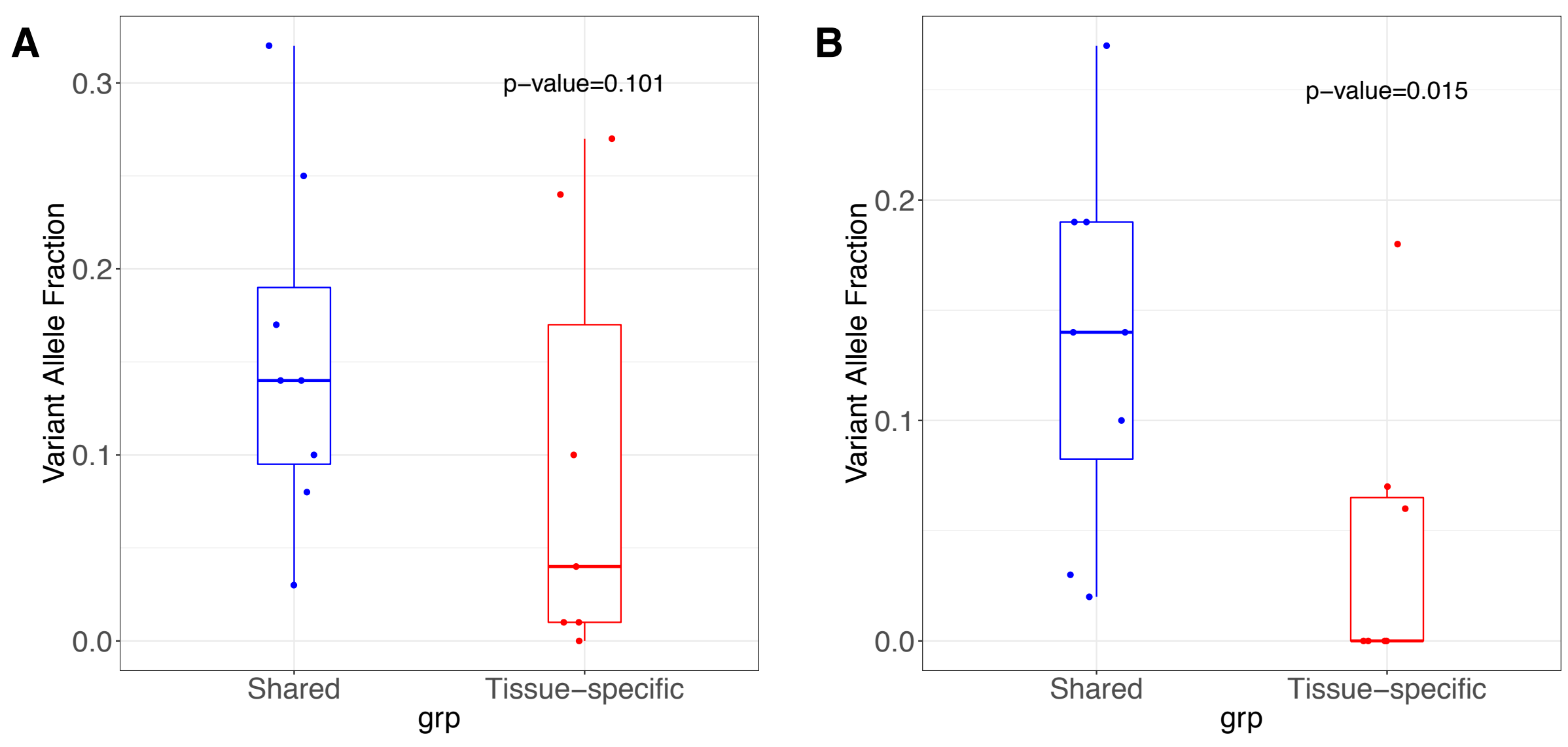


\section{Fig S10}

A

Likely Benign

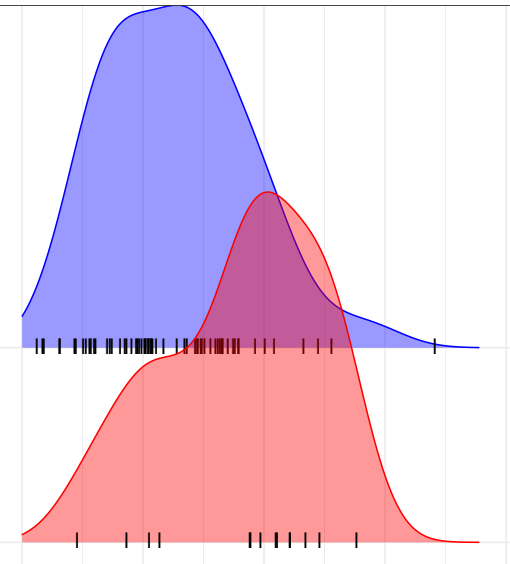

$p-$ value $=0.005$

B

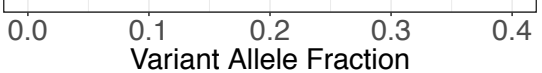

Likely Benign
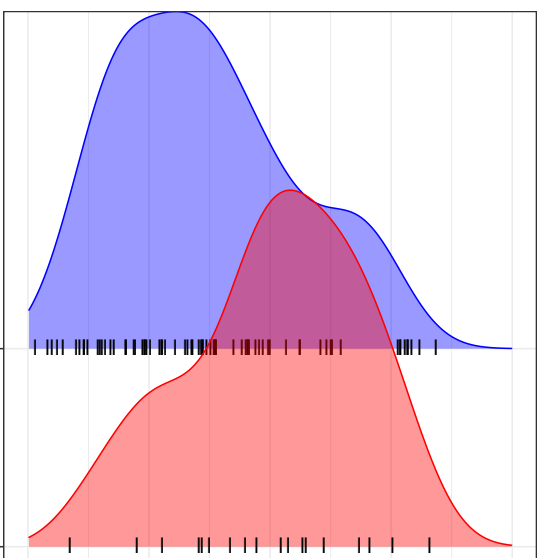

Damaging $p-$ value $=0.005$

$\begin{array}{llll}0.0 & 0.1 & 0.2 & 0.3\end{array}$
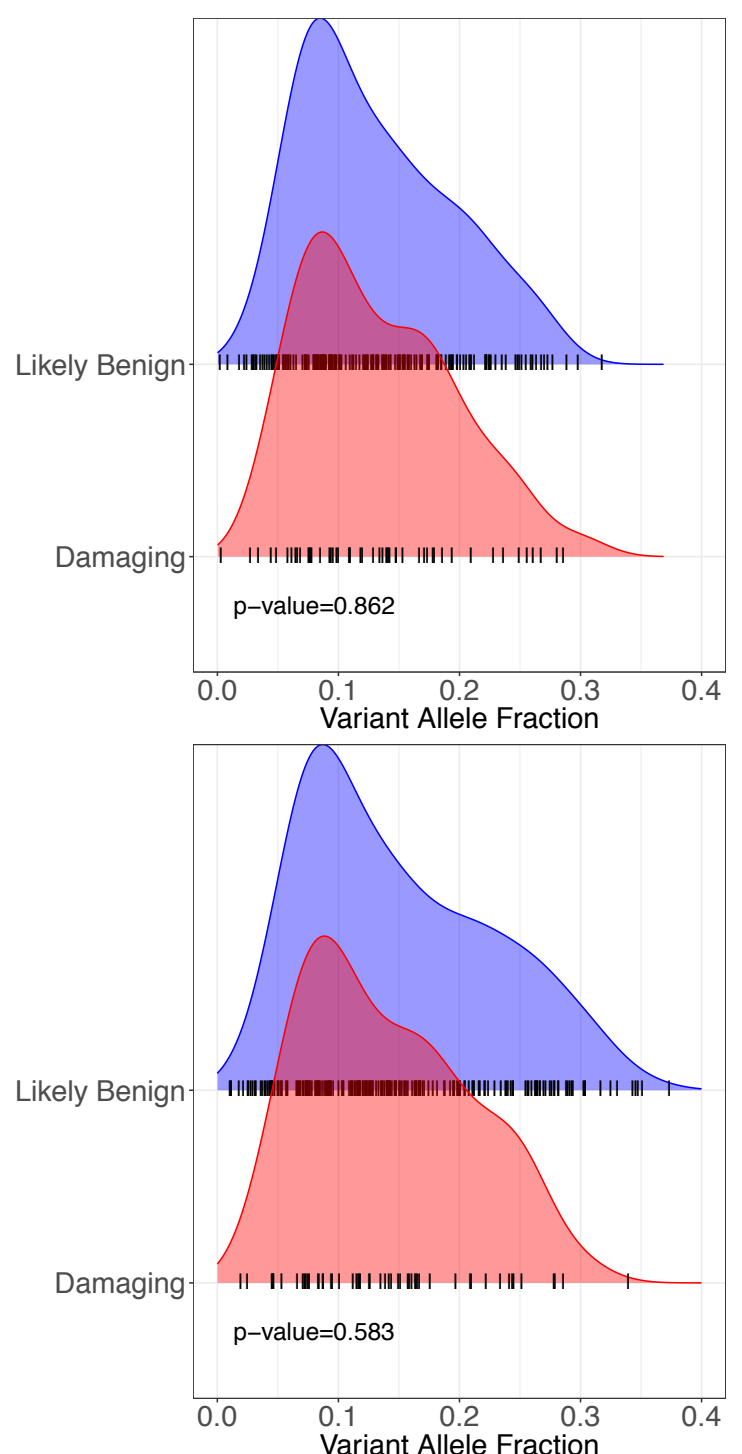


\section{Fig S11}

A

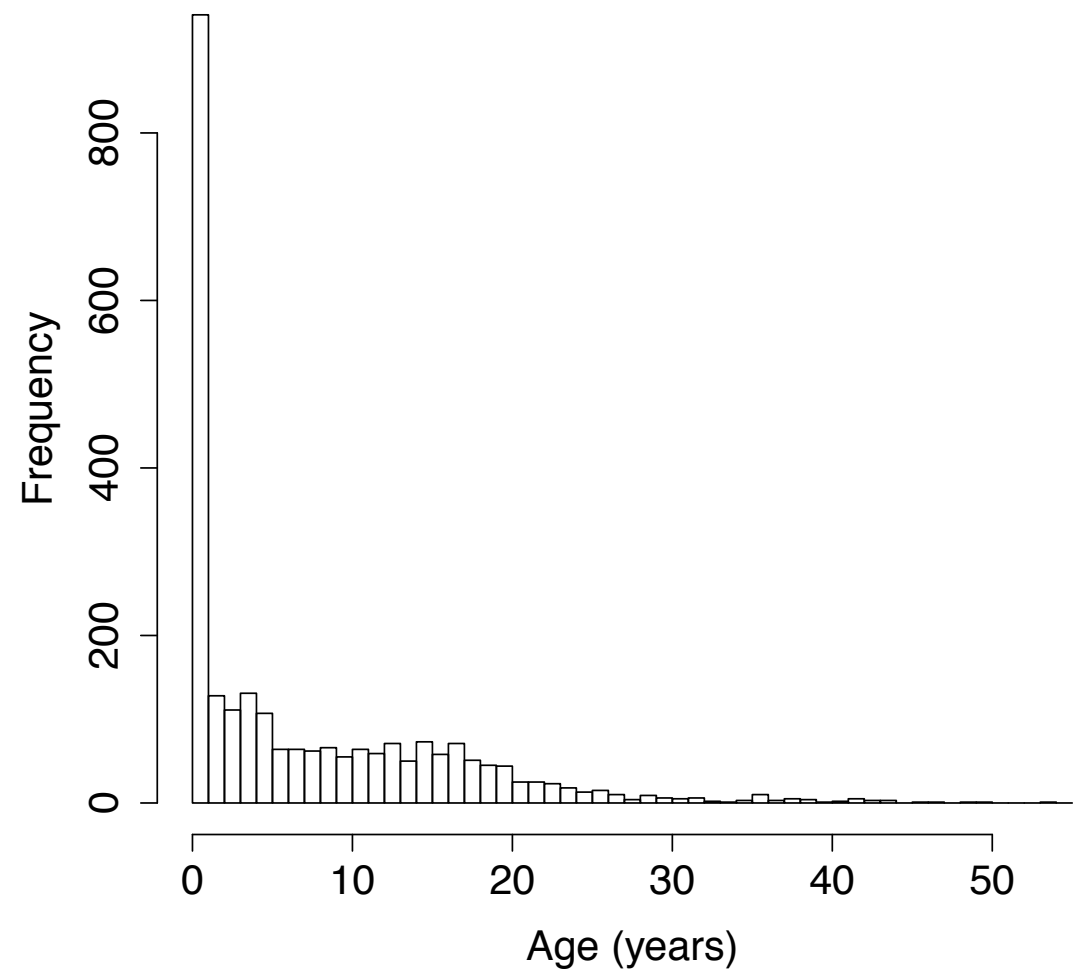

B

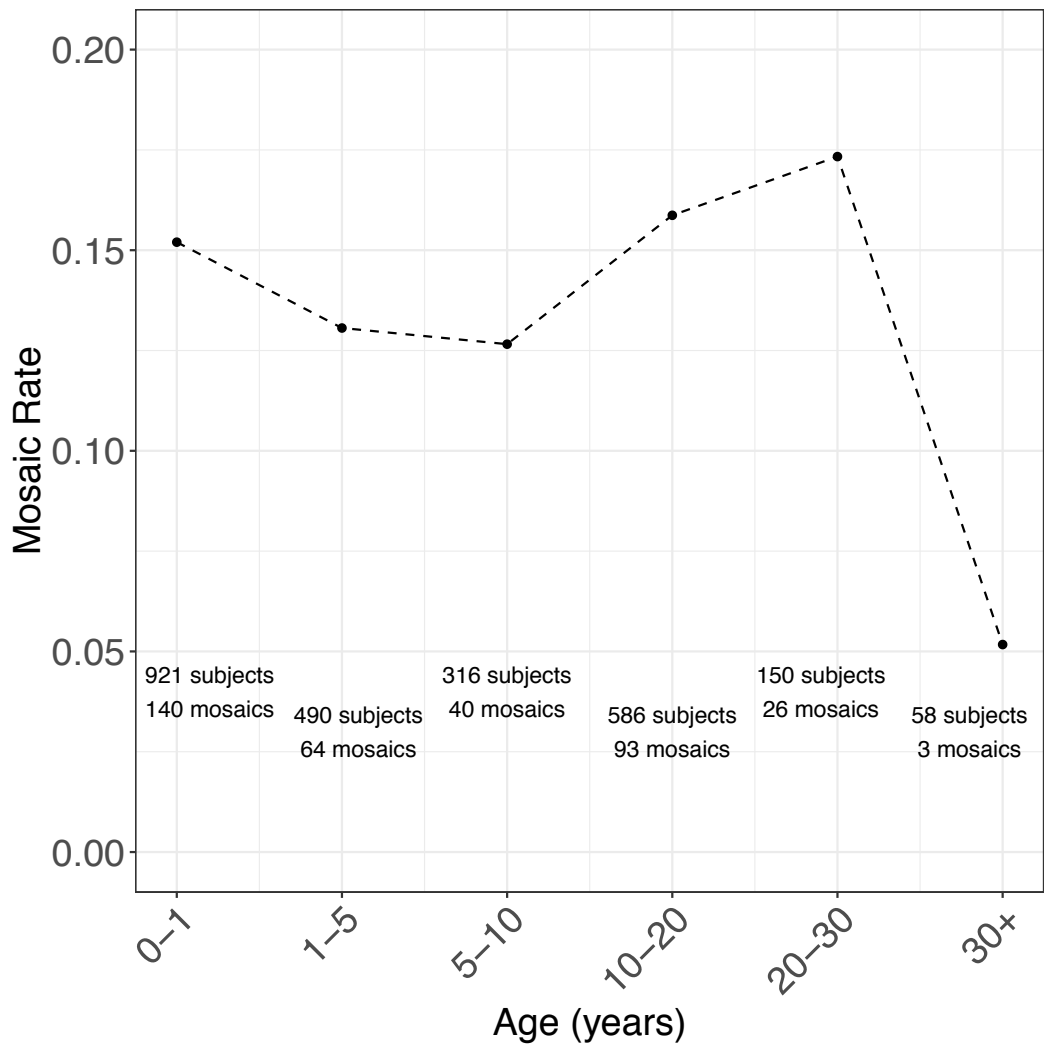




\section{Fig S12}

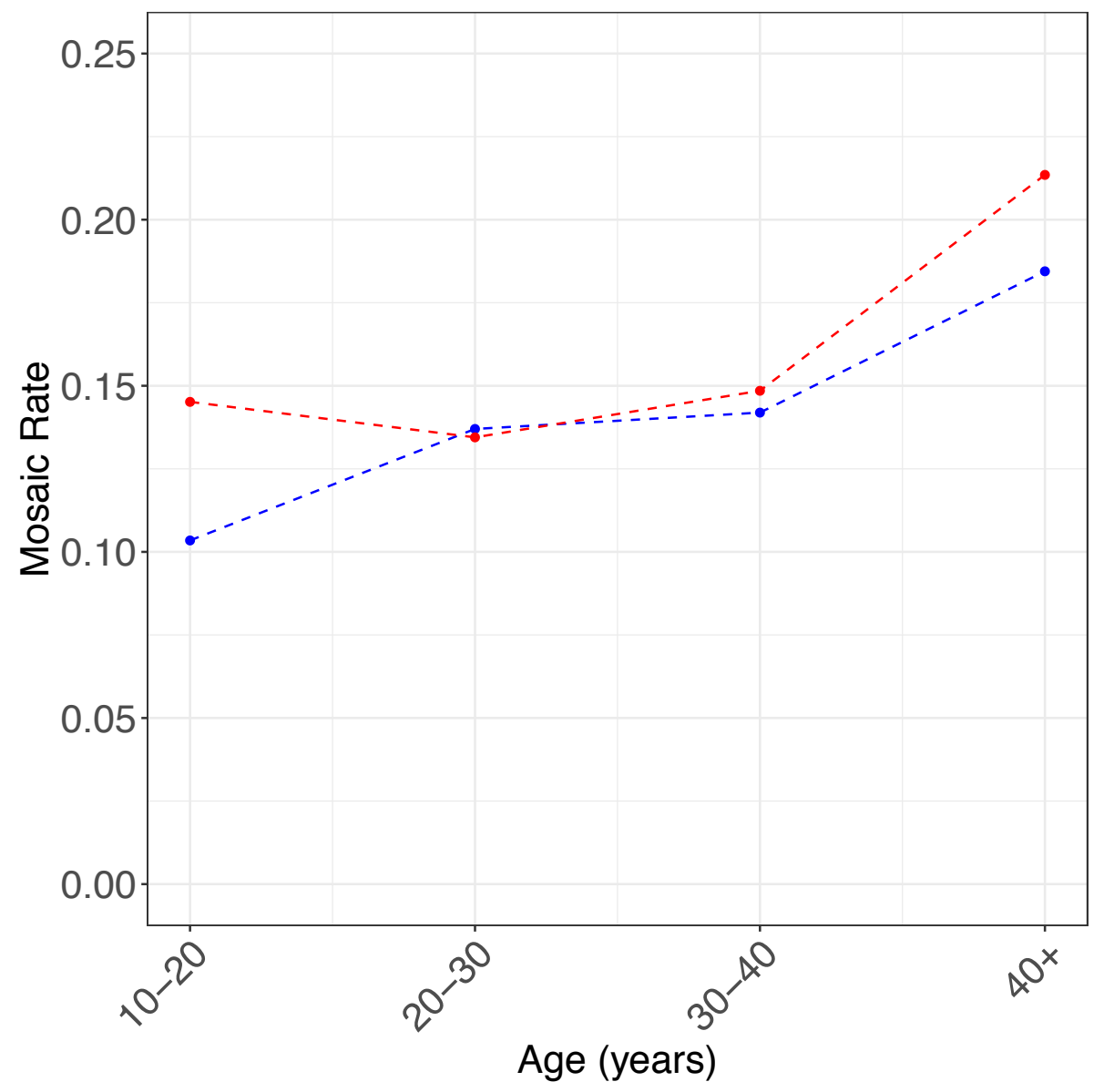


Fig S13
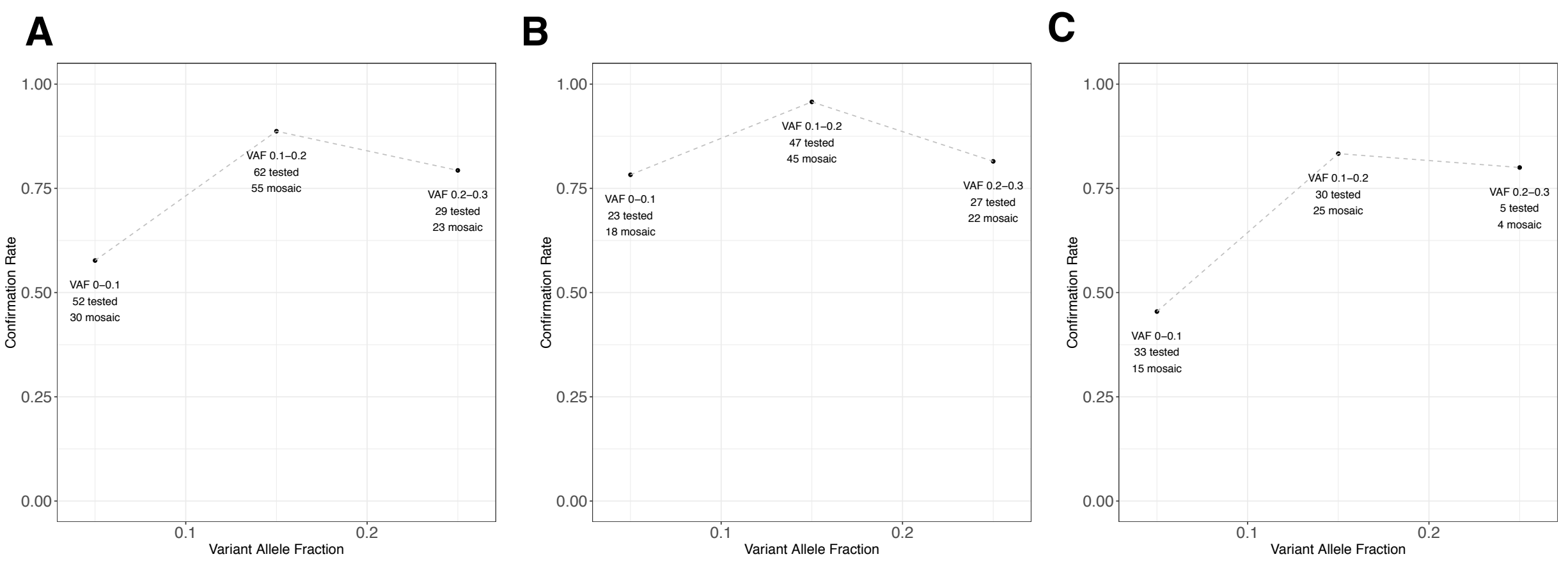
Fig S14

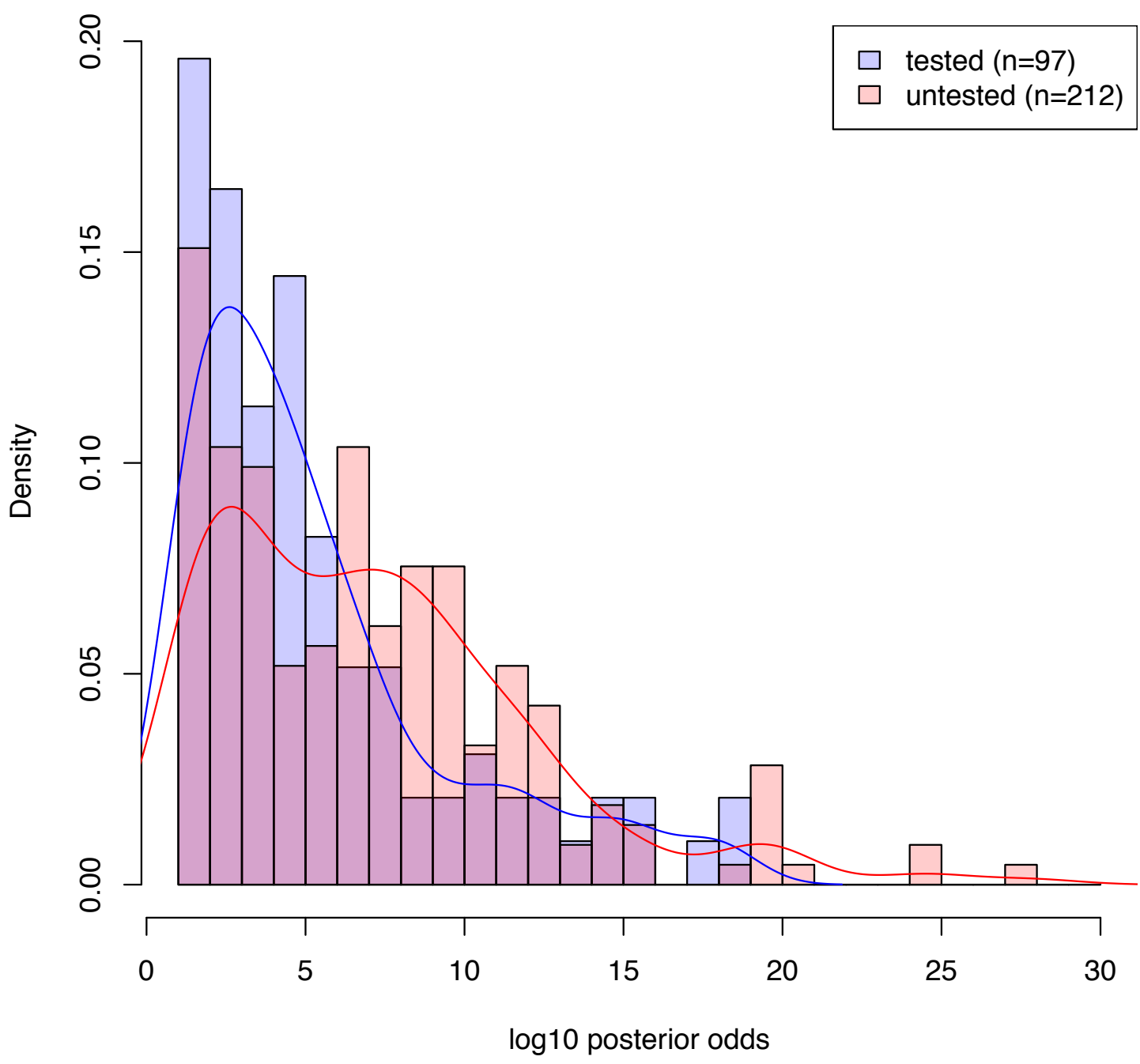

